\title{
Geochemistry of sericite deposits at the base of the Paleoproterozoic Aravalli Supergroup, Rajasthan, India: Evidence for metamorphosed and metasomatised Precambrian Paleosol
}

\author{
B Sreenivas, A B RoY* and R Srinivasan \\ National Geophysical Research Institute, Hyderabad 500007, India. Email: postmast@csngri.ren.nic.in \\ ${ }^{*}$ M L Sukhadia University, Udaipur 313002, India.
}

\begin{abstract}
Fine grained sericite deposits occur at the interface between Archean Mewar Gneiss Complex and the Proterozoic Aravalli Supergroup independent of shearing. They show a gradational contact with the basement granites and gneisses and a sharp contact with the overlying quartz pebble conglomeratic quartzites. Rip-up clasts of these sericite schists are found in the overlying conglomerates. The sericite schists are rich in sericite towards the top and contain chlorite towards the base. The sericite in these schists was formed by metasomatic alteration of kyanite and not from the feldspars of the basement granitoids and gneisses. Uni-directional variations of $\mathrm{SiO}_{2}$ and $\mathrm{Al}_{2} \mathrm{O}_{3}$, high $\mathrm{Al}_{2} \mathrm{O}_{3}$ content $(>30 \%$ ), positive correlation between $\mathrm{Al}_{2} \mathrm{O}_{3}$ and $\mathrm{TiO}_{2}, \mathrm{Ti} / \mathrm{Al}$ and $\mathrm{Ti} / \mathrm{Zr}$ ratios, high pre-metasomatic chemical indices of alteration $(>90)$, and enrichment of heavy rare earth elements relative to the parent granites and gneisses-all these chemical characteristics combined with field evidence suggest that the sericite schists are formed from a paleosol protolith, which developed on Archean basement between 2.5 and $\sim 2.1 \mathrm{Ga}$ in the Precambrian of Rajasthan. The superimposed metasomatic alteration restricts the use of $\mathrm{Fe}^{2+} / \mathrm{Ti}$ and $\mathrm{Fe}^{3+} / \mathrm{Ti}$ ratios of these paleosols for interpretation of $\mathrm{PO}_{2}$ conditions in the atmosphere.
\end{abstract}

\section{Introduction}

Paleosols are useful rock types for understanding the climatic conditions and atmospheric compositions of the geological past. Compositions of paleosols have been effectively used to estimate the $\mathrm{PO}_{2}$ of the atmosphere prevalent at the time of their development (Holland 1984; Holland and Zbinden 1988; Pinto and Holland 1988; Zbinden et al 1988; Feakes et al 1989; Holland et al 1989; Holland and Beukes 1990; Holland 1994; MacFarlane et al 1994; Ohmoto 1996; Rye and Holland 1998). In spite of this potential, the number of reported unambiguous Precambrian paleosols is relatively small. The reason for this is that many of the soil profiles of the distant geological past may have been eroded away, and it is very difficult to identify paleosols in the highly deformed and metamorphosed Precambrian terranes.
While deformation may affect the primary field relations, metamorphism obscures the primary soil textures because of recrystallization. Even in such cases, geochemical signatures have been found to be useful in identification of paleosols. However, it is of interest to note that application of geochemical signatures for identifying paleosols in highly deformed and metamorphosed terranes has led to a number of debates not only on their origin but also regarding their validity as good barometers of oxygen in atmosphere (Kallioski 1975, 1977; Lewan 1977; Barrientos and Selverstone 1987, 1988; Williams 1988; Palmer et al 1989; Ohmoto 1996, 1997; Holland and Rye 1997; Rye and Holland 1998). One of the main problems is how to distinguish the geochemical variations produced during subaerial weathering from those resulting from other alterations such as hydrothermal alterations in shear zones. There is no single

Keywords. Paleoproterozoic; Aravalli Supergroup; paleosols; geochemistry; weathering indices; trace and REE compositions. 
criterion that can independently distinguish the original nature of any metamorphic rock as a paleosol. An integrated approach comprising study of field relations, petrography and compositional variations may together possibly lead to identification of paleosols with greater confidence in deformed and metamorphosed Precambrian terranes. Identification of paleosols in such terranes is helpful in understanding stratigraphic relations (Barrientos and Selverstone 1988), sedimentary facies of the supracrustal sequence (Williams 1988) and crustal evolution in a particular metamorphosed terrane (Retallack 1990). Metamorphosed paleosols give at least a qualitative understanding of $\mathrm{PO}_{2}$ levels in the atmosphere of the past.

Study of paleosols in the Precrambrian sequences of India has so far received very little attention. Dash et al (1987) reported that khondalites of Orissa probably represent metamorphosed Precambrain aluminous lateritic soils. Similar high $\mathrm{Al}_{2} \mathrm{O}_{3}$ soils have been proposed as protoliths of the Precambrain sillimanite-corundum deposits of Sonapahar of Meghalaya state, northeastern India (Golani 1989). Some pyrophyllite-diaspore deposits of the Bundelkhand Complex of Central India have been interpreted as paleosols by Sharma (1979). In an attempt to chemically distinguish metamorphosed paleosols from metapelites of the Precambrian of peninsular India, Sreenivas and Srinivasan (1994) proposed that khondalites, especially of northern parts of the Eastern Ghat Mobile Belt may represent metamorphosed Precambrian paleosols, whereas some high alumina rocks of the Holenarasipur schist belt of the Dharwar craton are metamorphosed hydrothermal alteration products. Apart from these, large deposits of fine grained micaceous rock at the base of the Proterozoic Aravalli sequence (being mined as "pyrophyllite") have been interpreted by Roy and Paliwal (1981) as possible paleosols. A major element geochemical study by Banerjee (1996) has also suggested that these rocks may be Precambrian paleosols. However, some workers have considered that these rocks may represent products of shearing (Ahmad and Rajamani 1988) or hydrothermal alteration products of gneisses (Chauhan 1970). To understand their origin, much more detailed examination including trace and rare earth element geochemical studies are essential. In this work, we present a field geological, petrographic and geochemical account of these deposits from the Udaipur region of the Aravalli Mountain Belt and discuss their origin and significance.

\section{Geological setting}

The Archean basement for the Aravalli Supergroup, referred to as the Mewar Gneiss Complex (MGC, after Roy 1988; Roy and Kroner 1996) consists of biotite gneisses and granites with enclaves of amphibolites, quartzites and marbles. The rocks of the MGC range in age between 3.3 and 2.5 Ga (Gopalan et al 1990; Wiedenbeck et al 1996; Roy and Kroner 1996). The Aravalli Supergroup consisting of volcanic and sedimentary rocks unconformably overlies the granites and gneisses of the MGC. These are considered to be of $\sim 2.1 \mathrm{Ga}$ age based on a $\mathrm{Pb}-\mathrm{Pb}$ age of galena from the volcanic sequence in the lowermost part of the Aravalli Supergroup (Deb 1999). At the interface of the lowermost Aravalli Supergroup and the Archean MGC, fine grained mica deposits occur discontinuously and are being mined as "pyrophyllite". But wherever they occur, they are always at the stratigraphic contact between Archean gneisses/ granites of the MGC and the Aravalli Supergroup. This stratigraphic relation led Roy and Paliwal (1981) to consider these sericite schists as paleosols representing a weathering profile, which developed at the top of Archean granites and gneisses.

The mica deposits (referred to as sericite schists hereafter) have been examined in detail from three localities near Udaipur:

- Tulsi-Namla area,

- Barodia quarry, and

- Madar (figure 1).

In the Tulsi-Namla area, the sericite schists dip beneath the Archean basement. Here they occur in the inverted limb of a steeply dipping isoclinal fold involving both the basement and cover. In the Barodia area the sericite schists occur between the quartz pebble conglomerate of the Aravalli Supergroup and the Ahar River Granite which is shown to be a part of the $>2.5$ Ga MGC (Wiedenbeck et al 1996). The rock formations here are strongly sheared. While the sericite schist shows folding, the granites close to the contact show strong brittle to brittleductile deformation. Near Madar, a normal stratigraphic relation has been observed between the Ahar River Granite and the sericite schist. No shearing at the contact is evident here. The sericite schist bodies are generally thin, their thickness varying from 2 to $12 \mathrm{~m}$. The contact of the sericite schists is gradational with the underlying gneiss but is sharp with the Aravalli quartzites or conglomerates that overlie them. Close to the upper contact the rock is highly sericitic. Underlying this section is a layer where sericite is associated with quartz, chlorite, biotite and magnetite. Many of these mica deposits exhibit variations in color. In the lower part nearer to the basement gneiss/granite, the rock is brownish green and towards the upper contact it attains a light greenish white color. A typical section at Barodia quarry is illustrated in figure 2. At some places such as near Bari Lake, clasts of sericite schists are found in the overlying conglomeratic quartzites. 


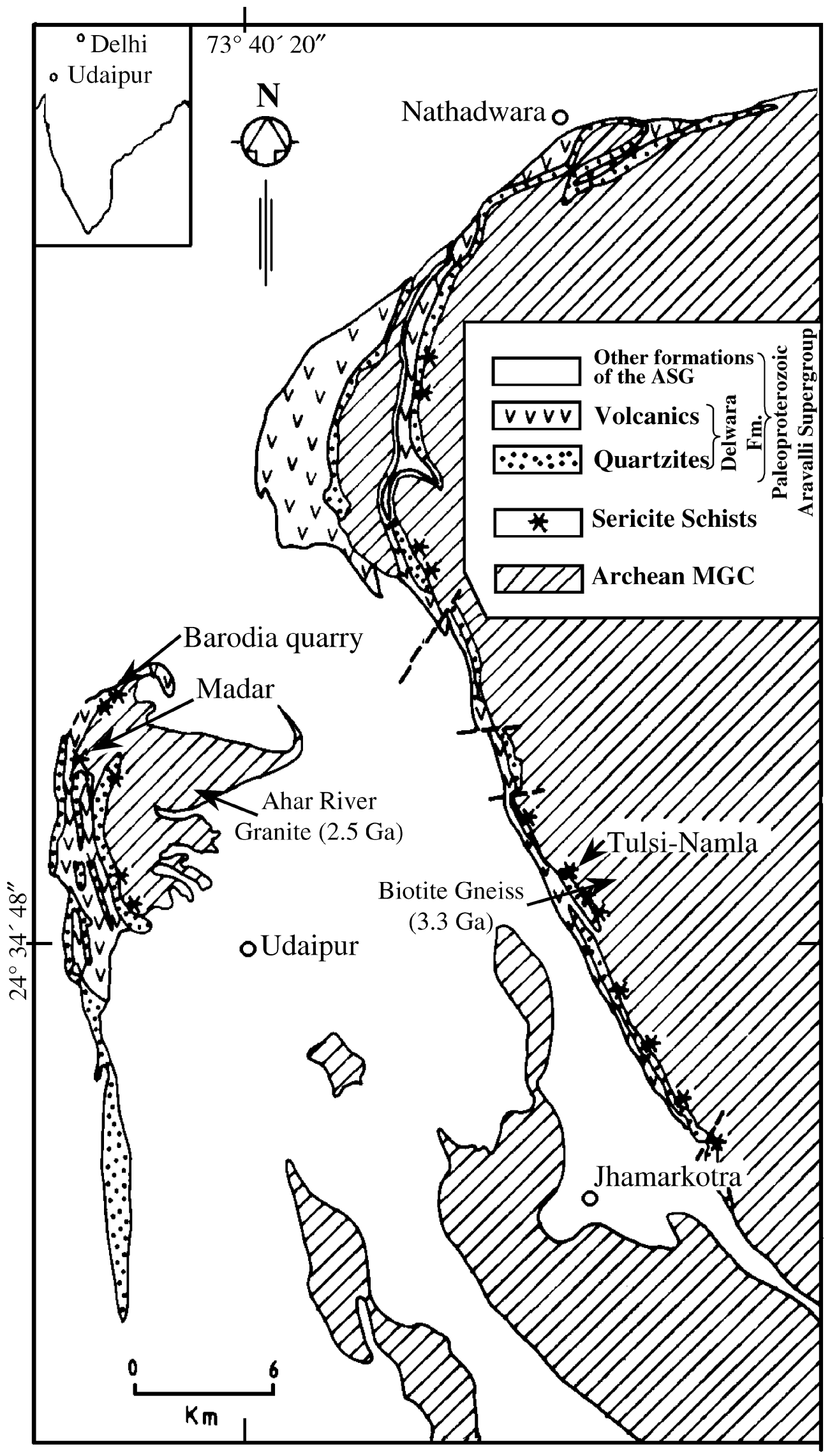

Figure 1. Geological map of the Udaipur-Nathadwara region, Rajasthan (northwestern India) showing occurrences of finegrained mica deposits at the stratigraphic interface between the Aravalli Supergroup and the Archean Mewar Gneissic Complex. Sample locations are also shown. 


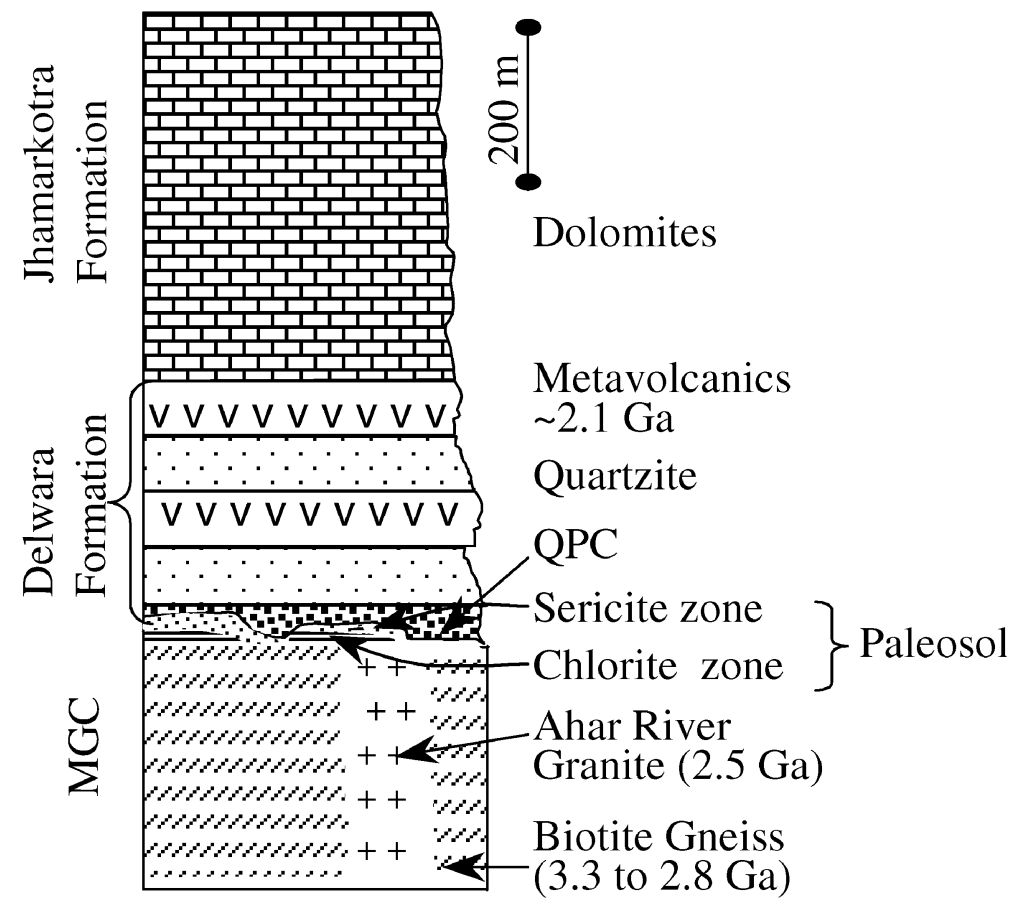

Figure 2. Typical section, illustrating the occurrence of sericite deposits near Barodia quarry. Note that there is an upper sericite and lower chlorite-quartz-sericite zone.

\subsection{Sampling}

A complete section from the basement granite up to the upper contact of the sericite schist with the overlying Aravalli quartzite is exposed in the quarry near Barodia. Here serial sampling has been carried out. Two distinct zones can be identified here consisting of lower chlorite rich and upper sericitic horizons. While the lower chlorite horizon is brownish in color, the upper sericite schist is pale green in color. In the Tulsi-Namla and Madar areas such a complete section of sericite and chlorite zones is not exposed. This made only cluster sampling possible in the latter two localities. Throughout the discussion on petrography and geochemistry, special emphasis is therefore given for the data obtained on samples from Barodia.

\section{Petrography}

\subsection{Sericite schists}

The sericite schist shows well developed schistosity at places and is massive elsewhere. Puckering on schistosity is common. A careful study of outcrops as well as hand specimens shows that the micaceous rock contains blades of kyanite. Thin section study reveals that the mica in these rocks replaces kyanite along cleavages and fractures (figure 3a). Larger relicts of kyanite measuring up to $1 \mathrm{~cm}$ have been observed especially in the samples of Tulsi-Namla area, while a more advanced stage of replacement by mica has left only tiny relicts of kyanite a few microns in size in the samples of the Madar area (figure $3 \mathrm{~b}$ ). The highest degree of replacement by white mica is in the samples of Barodia quarry where most samples have only small amounts of relict kyanites. Petrographic studies clearly demonstrate that the finegrained sericitic mica in these rocks originated due to the breakdown of kyanite by metasomatic alteration. The serial samples of Barodia exhibit mineralogical variations from the bottom to the top. The lower chlorite zone samples contain quartz-sericite-chlorite with rare relicts of kyanites. As noted earlier by Banerjee (1996) there are relict textures of basement granite in this zone. The sample that is near to the upper contact with the quartz pebble conglomerate is virtually free of quartz and is composed wholly of sericite. Thus excellent uni-directional variations in mineral assemblage appears to be characteristic of these sericite schists.

The sericite schists in addition to mica and kyanite, contain small amounts of zircon and rarely rutile. Two varieties of zircons have been noticed in the sericite schists. They are:

- reddish brown to hyacinth red colored, short and stumpy zircon crystals with double terminations (figure 3c), and,

- pale hyacinth red to olive green colored, transparent, long, prismatic zircons with double termination.

All the zircons are euhedral and show no evidence of rounding. The grains of rutile are blood red color and have a long prismatic habit. 


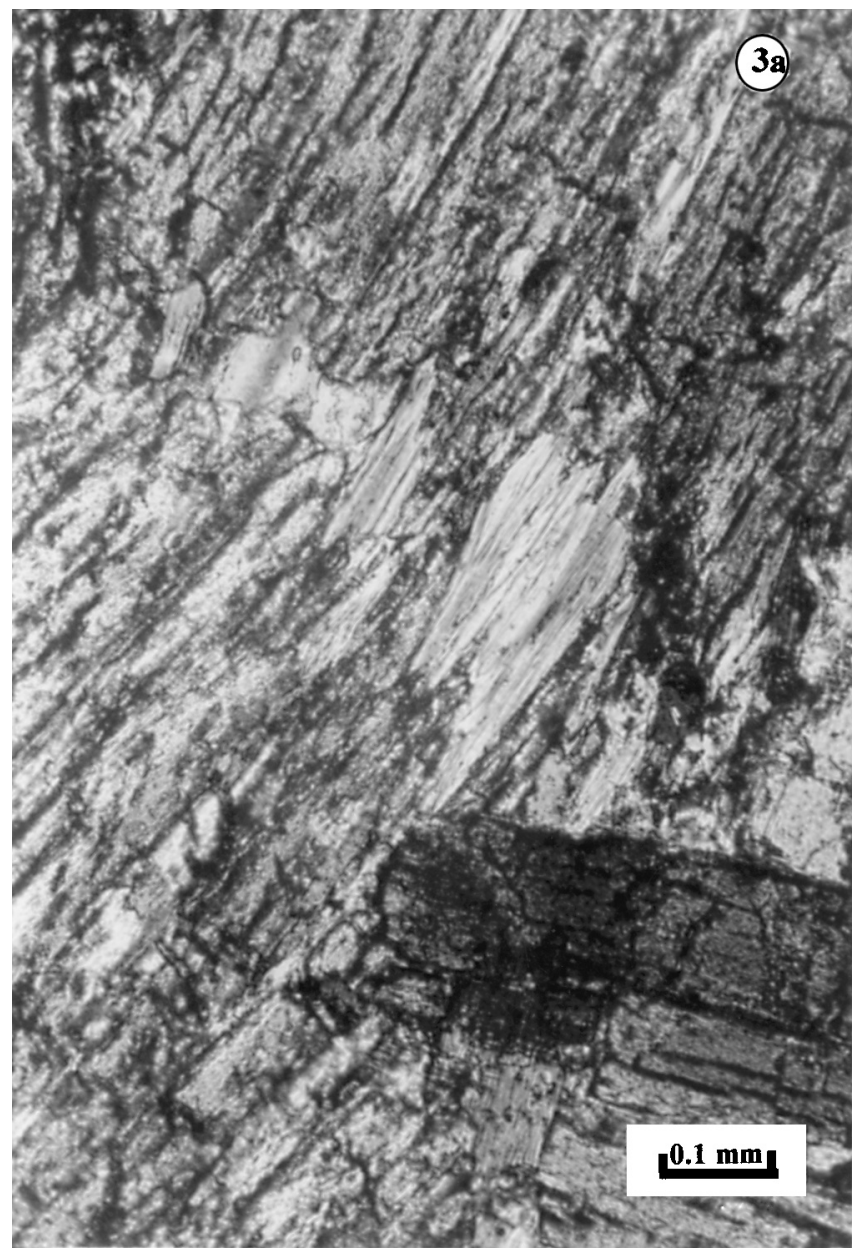

Figure 3. (a) Sericite replacing kyanite along cleavages and fractures-incipient stage, Tulsi-Namla area.

\subsection{Basement granite and gneiss}

The petrography and geochemistry of Ahar River Granite, which is the basement rock for sericite schists has been investigated earlier by Roy et al (1985), Rahman and Zainuddin (1990), and, Guha and Garkhal (1993). These earlier studies, as well as the observations during the present work, show that the Ahar River Granite is granite-gneiss complex which shows variation in mineralogical and chemical composition. It consists of a tonalite phase and a granodiorite phase. The tonalitic rocks are grey in color and are composed of oligoclase to andesine plagioclase, microcline, quartz and minor amounts of biotite. The plagioclase is saussuritized as represented by mineral assemblage sericite-epidote-chloritecalcite. Apatite, sphene and zircon are found as accessories. Guha and Garkhal (1993) recorded the occurrence of rutiles among the accessories. The zircons are euhedral, usually reddish brown in color, highly fractured, short and stumpy with double terminations (figure 4a). However, there are also other clean zircons, which are not fractured, long and zoned with double terminations. Some of these latter zircons have reddish brown cores on which there are pale hyacinth red colored, zoned overgrowths. Wiedenbeck et al (1996) have recognized two populations of $\mathrm{Pb}-\mathrm{Pb}$ zircon ages in the Ahar River Granite. An older one of $2.856 \mathrm{Ga} \mathrm{Pb}-\mathrm{Pb}$ isotopic age considered to be xenocrystic zircon and a younger population interpreted as magmatic zircons of $\sim 2.5 \mathrm{Ga}$ age. The reddish brown, fractured zircons noticed by us in the Ahar River Granite may correspond to the above mentioned xenocrystic component.

The granodioritic phase of Ahar River Granite is pink in color and consists of oligoclase, microcline, quartz and muscovite with biotite as the principal accessory. While plagioclase is strongly saussuritized the microcline has remained largely unaltered. Sphene, apatite, magnetite and zircon are the accessories. Zircons are euhedral, pale hyacinth red in color and show double terminations.

The biotite gneiss which underlies the sericite schists near Tulsi-Namla is a banded gneiss. The felsic bands alternate with bands rich in biotite. The felsic bands are composed of oligoclase and minor amounts of microcline. Biotite is the principal accessory. Plagioclase feldspars are generally untwinned and are saussuritized. Microcline however is fresh. Apatite, zircon and sphene are accessories. 


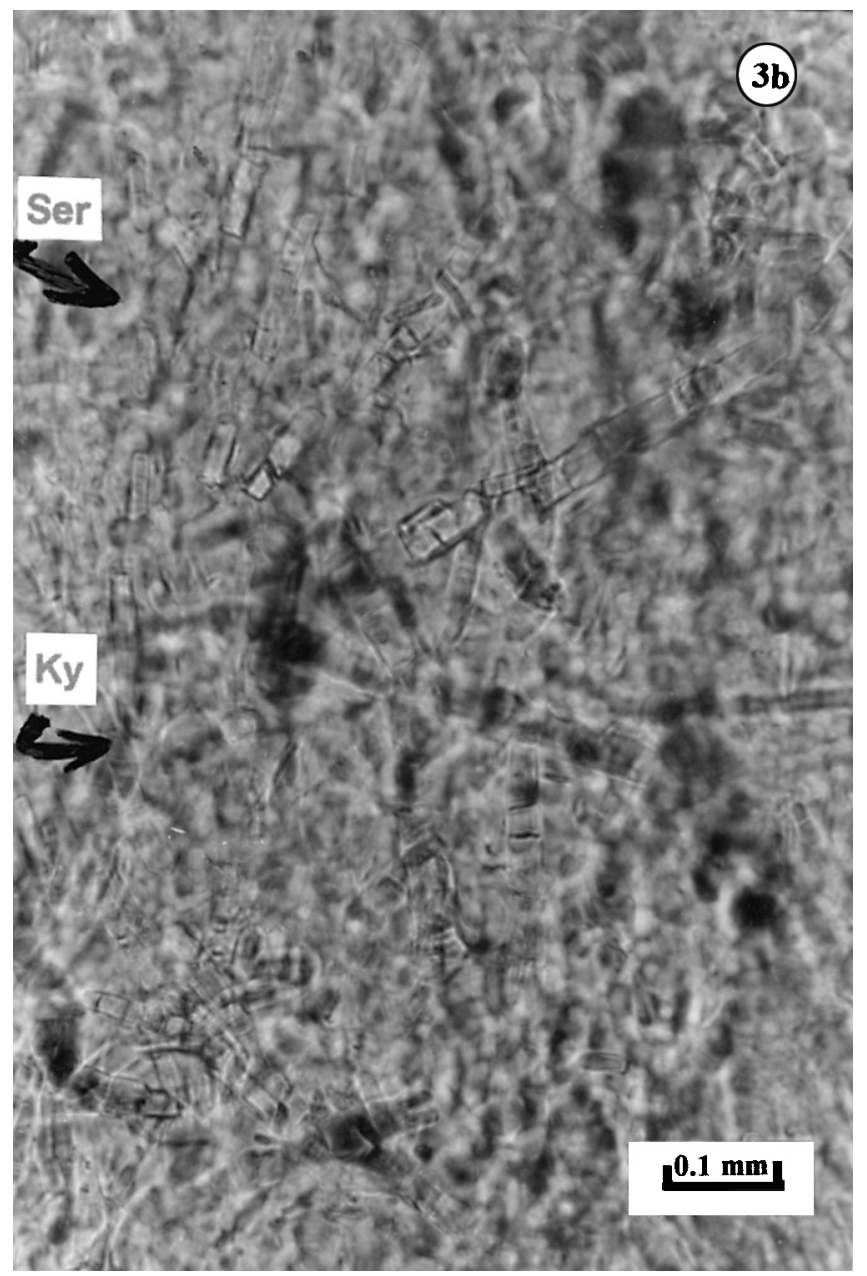

Figure 3. (b) Advanced stage of replacement of kyanite by sericite with tiny relicts of kyanite left in a dominantly sericitic matrix, Madar area.

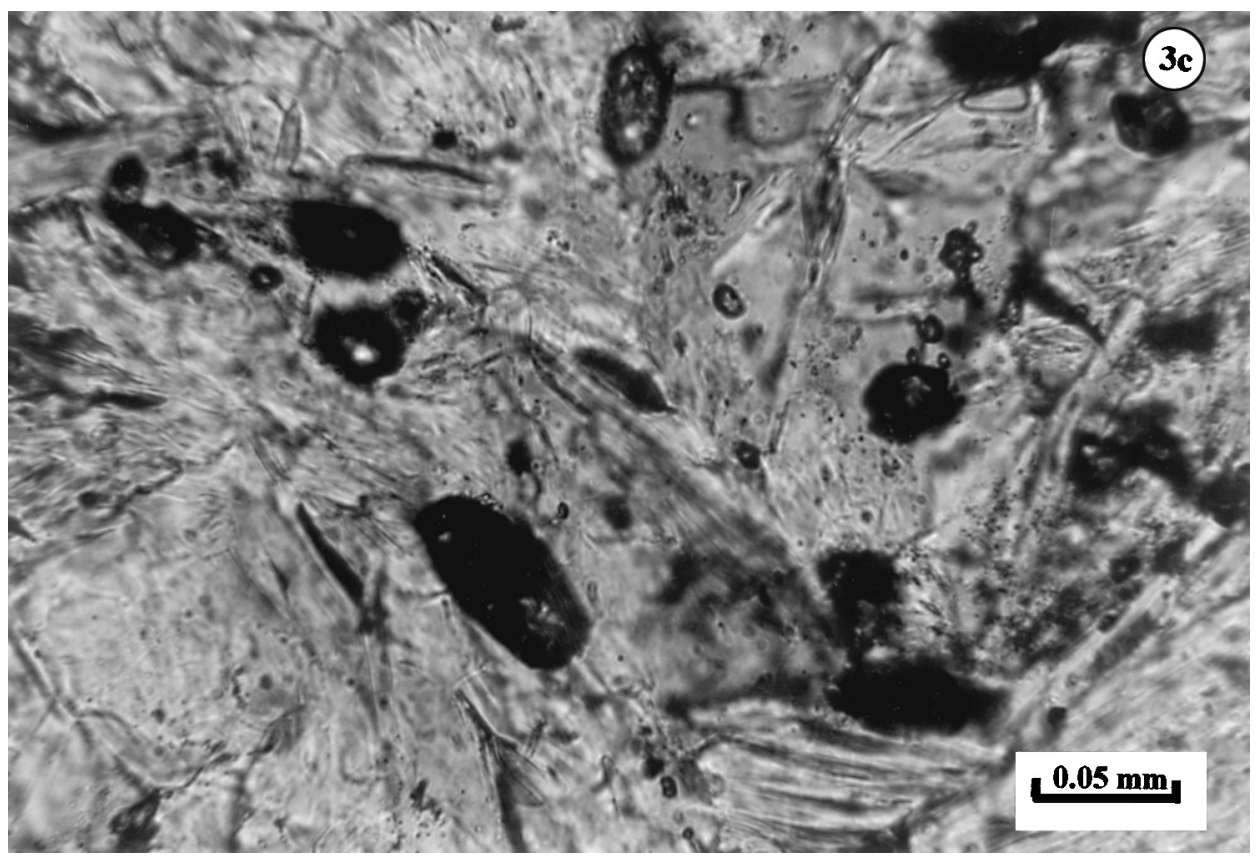

Figure 3. (c) Zircons in sericite schists, Madar area showing reddish brown as well as olive colored zircons usually short and stumpy with double terminations. 


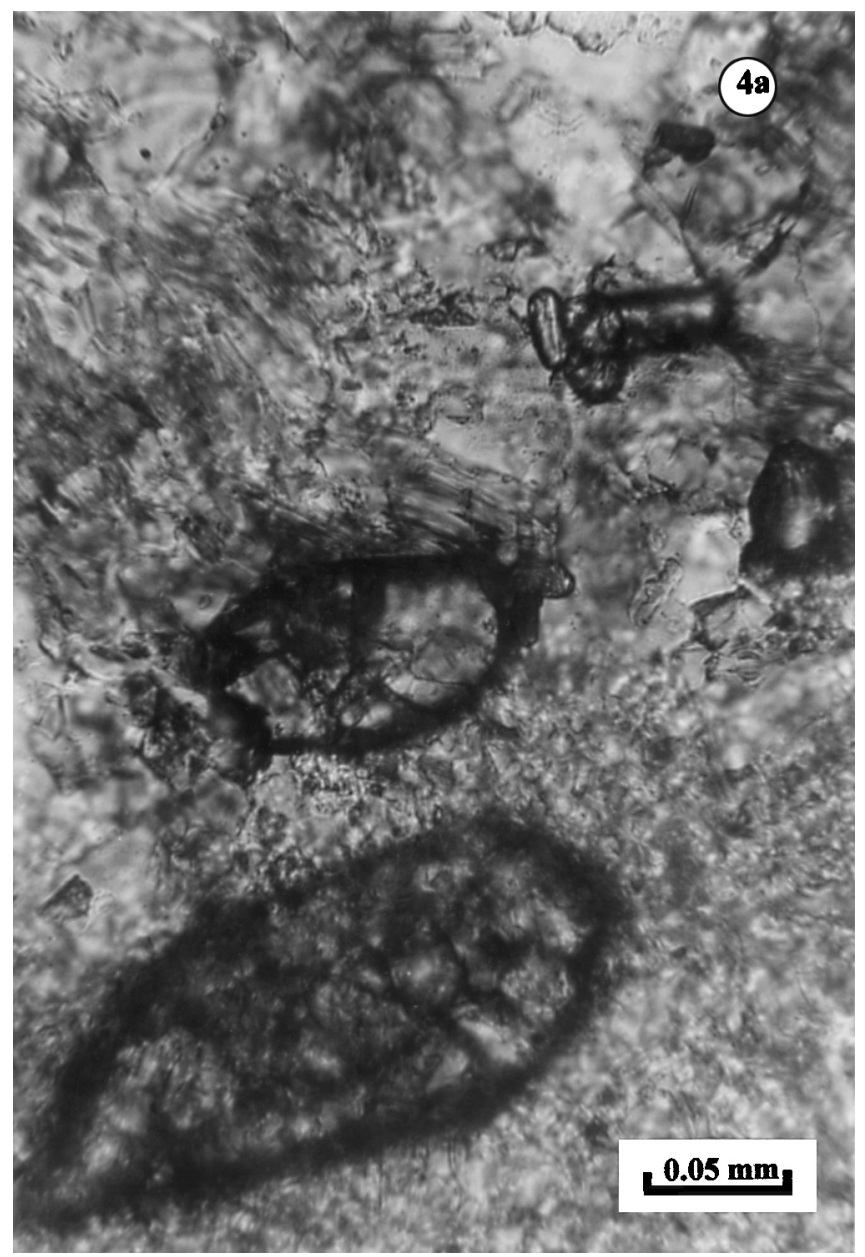

Figure 4. (a) Reddish brown, doubly terminated zircons as well as long, prismatic zircons in the Ahar River Granite. Note the gross similarity between these zircons and those presented in figure $3(\mathrm{c})$.

Zircons are seen to be more abundant in bands richer in biotite. The zircons are olive colored, show excellent zoning with olive colored core and brownish rim. All these zircons are doubly terminated (figure $4 \mathrm{~b}$ ).

\section{Geochemistry}

\subsection{Analytical techniques}

Analysis of individual mineral phases was performed on well polished thin sections using Camebax - micro WDS electron probe micro analyser (EPMA). Operating conditions were: accelerating voltage - $15 \mathrm{KV}$; beam current -4 to $6 \mathrm{nA}$; beam diameter - 1 to $5 \mu \mathrm{m}$; counting time - 10 seconds. Suitable natural and synthetic standards were used for on-line ZAF corrections following the procedure of Bence and Albee (1968). Care has been taken to analyze grains which are at least $\geq 5 \mu \mathrm{m}$ size (figure $4 \mathrm{c}$ ) to avoid inaccurate beam focusing.

For whole-rock chemical analysis fresh chips from rock samples were ultrasonically vibrated in de- ionized water and air dried. The samples were reduced to sand size in a tungsten carbide vial by crushing for 5 seconds in a Herzog swing-grind mill. Subsequently the samples were powdered to - 200 mesh using an agate mortar and pestle. This method is found to be appropriate for overcoming contamination that may arise during sample powder preparation (Sreenivas et al 1994). The Co content is not reported as the samples were crushed to sand size using a tungsten carbide mill.

Major elements as well as some trace elements ( $\mathrm{Cr}$ and $\mathrm{Zr}$ ) were determined by a sequential X-ray fluorescence spectrometer (XRF) (Philips PW 1400 $\mathrm{XRF}$ ). Fused beads of the samples were used for major element analyses while pressed pellets were used for analyzing $\mathrm{Na}, \mathrm{Cr}$ and $\mathrm{Zr}$. $\mathrm{Na}_{2} \mathrm{O}$ and $\mathrm{K}_{2} \mathrm{O}$ were also measured by atomic absorption spectrometry (AAS). Synthetic standards covering the compositional range of the sericite schists were prepared from spec pure chemicals. The accuracy and precision of the data calculated using the $\chi^{2}$ method on these samples is well within acceptable limits (Sreenivas and Govil 1997). The $\mathrm{K}_{2} \mathrm{O}$ values obtained by both XRF and 


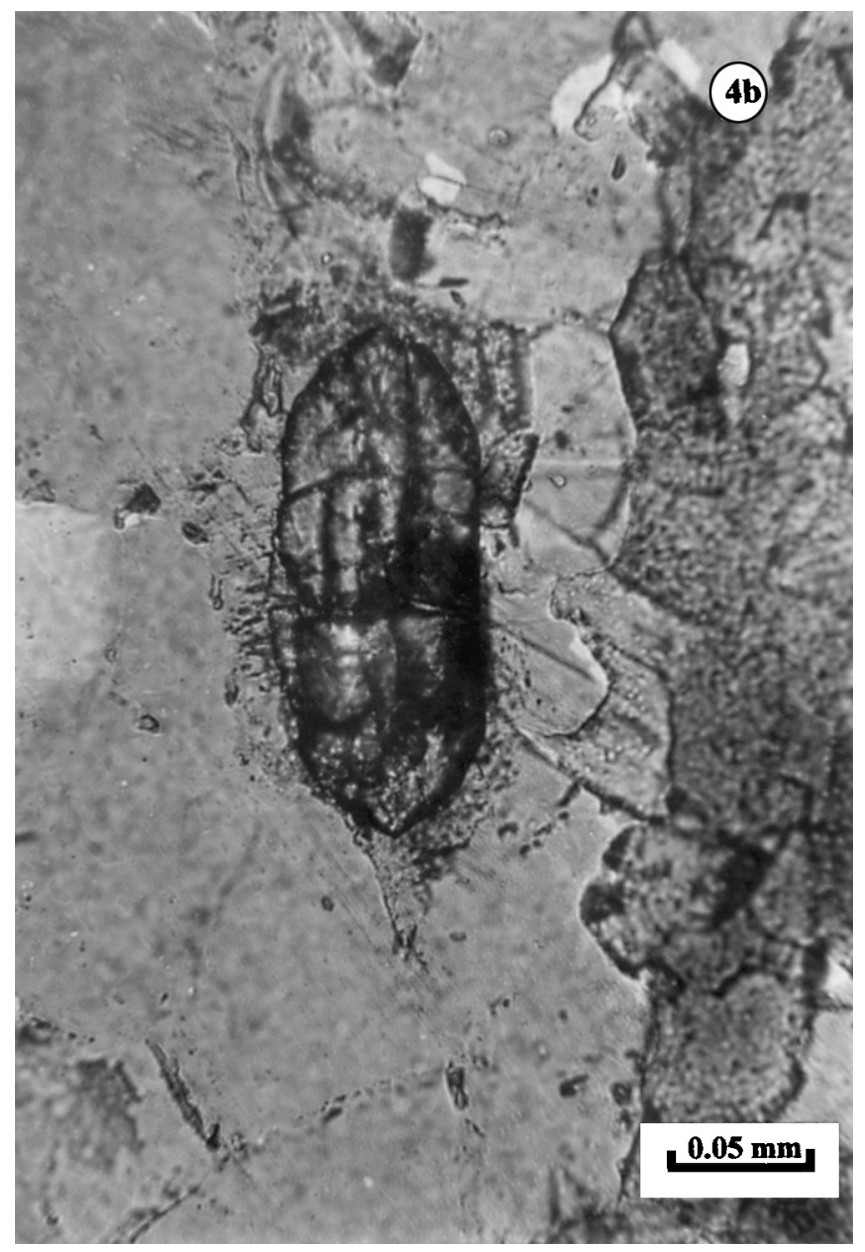

Figure 4. (b) Doubly terminated olive colored zircons in biotite gneiss of Tulsi-Namla.

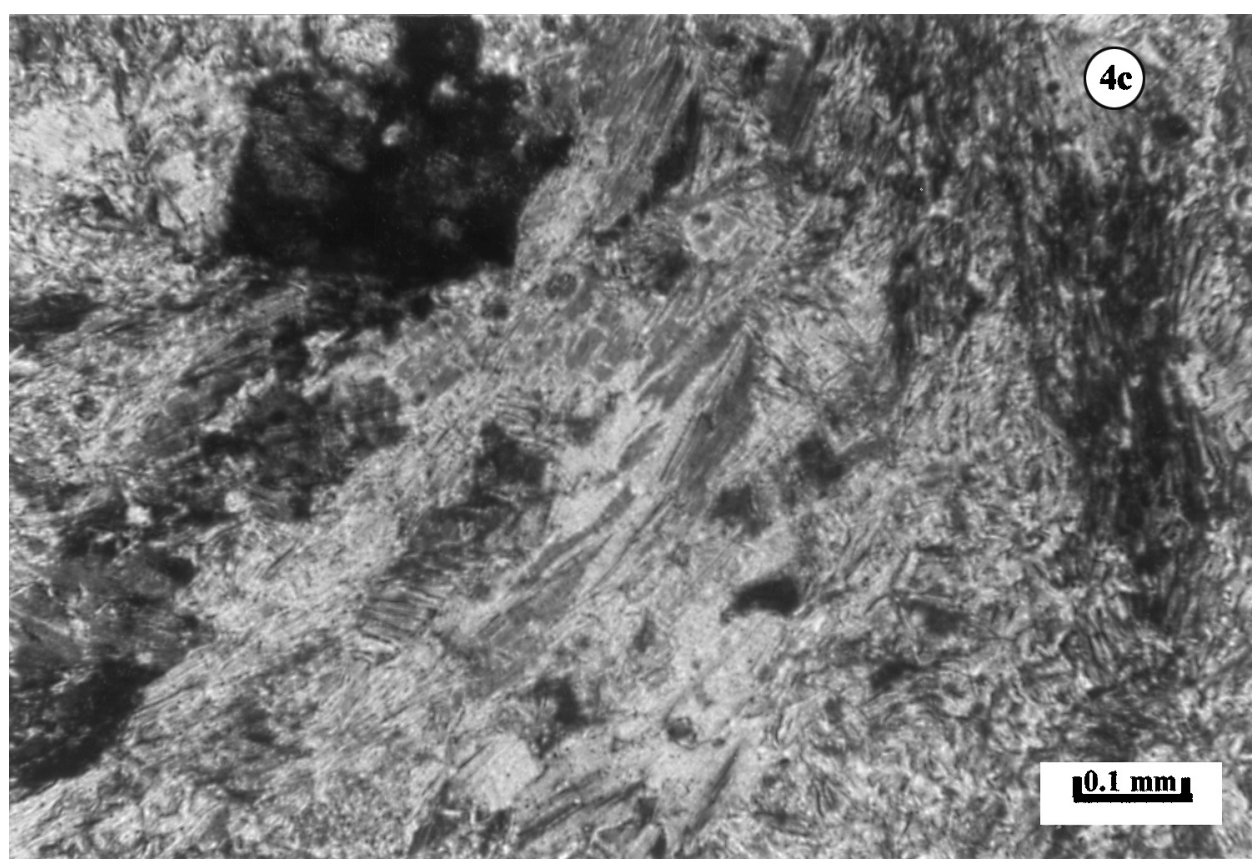

Figure 4. (c) Sericite schist of Madar area showing variable grain sizes of mica. Coarse grained portions are the ones where microprobe analyses have been carried out. 
AAS methods are in very good agreement with each other.

Eighteen trace and 11 rare earth elements were determined by ICP-MS. For this purpose the samples were taken into solution in an acid mixture comprising $7 \mathrm{ml} \mathrm{48 \%}$ EL grade $\mathrm{HF}, 3 \mathrm{ml}$ of concentrated $\mathrm{HNO}_{3}$ and $1 \mathrm{ml}$ of perchloric acid. The acid solutions were then heated at $200^{\circ} \mathrm{C}$ until the contents were evaporated to dryness. Then the residues were dissolved in $1: 1 \mathrm{HNO}_{3}$ and the volume increased to $100 \mathrm{ml}$ with MQ water. Solutions of international reference standards (G-2 granite; W-2 diabase USGS standards; BX-N French bauxite standard; NIM-S South African syenite standard; JF-1, JF-2 GSJ Japanese feldspar standards; MRG-1 Canadian gabbro standard; GSR-4 Chinese sandstone standard) and procedural blanks were also prepared in the same manner. The data obtained on the international reference standards that were analyzed during the same runs as the unknown samples show that the accuracy of the determinations are better than $95 \%$. Further details of analytical procedures are available in Balaram et al (1996).

\subsection{Mineral composition}

Compositions of kyanite, mica and rutile in sericite schists and of feldspars and micas of the underlying basement gneisses are presented in table 1. The mica in the schistose rocks is essentially $\mathrm{K}_{2} \mathrm{O}$-rich (c.10\%) indicating that they are not pyrophyllitic but sericitic/muscovitic in composition. The samples from Tulsi-Namla and Barodia quarries are dominated by mica with high $\mathrm{K}_{2} \mathrm{O}$ contents (c.10\%), while those from the Madar area have similar mica, and in addition, two other types which occur in microscopic patches. One variety has only $\sim 0.05 \% \mathrm{~K}_{2} \mathrm{O}$, which corresponds in composition to pyrophyllite. The other has $7 \% \mathrm{~K}_{2} \mathrm{O}$ and has composition of hydromuscovite. The sericite/muscovite replacing kyanite has 6 to $7 \%$ more $\mathrm{Al}_{2} \mathrm{O}_{3}$ than the sericite formed by the alteration of feldspars in the basement gneiss (figure 5a). The

Table 1. Average compositions of the minerals in the sericitic rocks and basement gneisses at their contact.

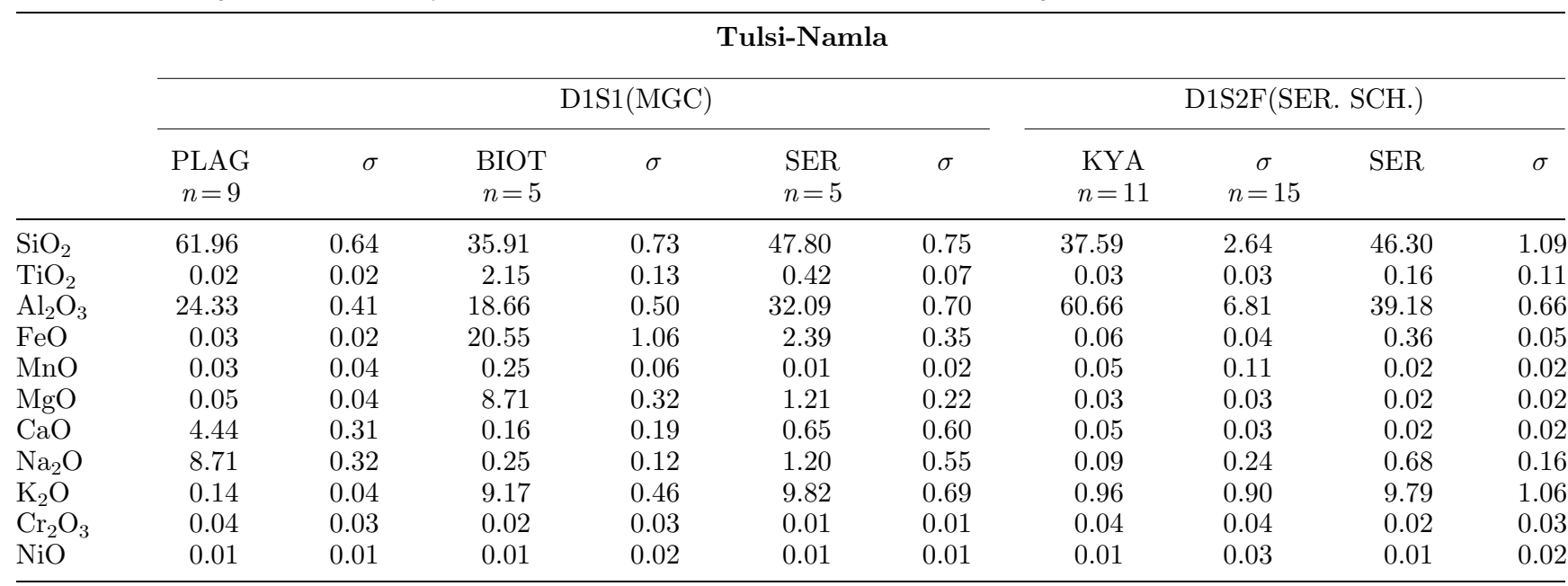

Madar

G4/1 (SER. SCH.)

\begin{tabular}{|c|c|c|c|c|c|c|c|c|c|c|c|c|}
\hline & \multicolumn{8}{|c|}{ G4/1 (SER. SCH.) } & \multicolumn{4}{|c|}{ W4/6 (SER. SCH.) } \\
\hline & $\begin{array}{l}\text { KYA } \\
n=22\end{array}$ & $\sigma$ & $\begin{array}{c}\text { SER } \\
n=11\end{array}$ & $\sigma$ & $\begin{array}{c}\text { HYDMUS } \\
n=2\end{array}$ & $\sigma$ & $\begin{array}{c}\text { PYRO } \\
n=9\end{array}$ & $\sigma$ & $\begin{array}{l}\text { SER } \\
n=9\end{array}$ & $\sigma$ & $\begin{array}{l}\text { RUT } \\
n=5\end{array}$ & $\sigma$ \\
\hline $\mathrm{SiO}_{2}$ & 36.92 & 0.50 & 46.35 & 0.56 & 51.76 & 0.33 & 64.92 & 1.22 & 46.02 & 0.60 & 0.43 & 0.23 \\
\hline $\mathrm{TiO}_{2}$ & 0.03 & 0.04 & 0.05 & 0.03 & 0.04 & 0.03 & 0.02 & 0.02 & 0.08 & 0.04 & 97.57 & 0.85 \\
\hline $\mathrm{Al}_{2} \mathrm{O}_{3}$ & 62.61 & 0.76 & 38.34 & 0.36 & 35.99 & 1.07 & 29.13 & 1.46 & 38.03 & 0.45 & 0.23 & 0.12 \\
\hline $\mathrm{FeO}$ & 0.25 & 0.06 & 1.14 & 0.22 & 0.99 & 0.38 & 0.14 & 0.06 & 0.45 & 0.11 & 0.61 & 0.10 \\
\hline $\mathrm{MnO}$ & 0.02 & 0.02 & 0.03 & 0.03 & 0.01 & 0.01 & 0.01 & 0.02 & 0.02 & 0.02 & 0.01 & 0.01 \\
\hline $\mathrm{MgO}$ & 0.02 & 0.02 & 0.09 & 0.05 & 0.07 & 0.01 & 0.03 & 0.02 & 0.17 & 0.13 & 0.01 & 0.01 \\
\hline $\mathrm{CaO}$ & 0.03 & 0.02 & 0.00 & 0.00 & 0.00 & 0.00 & 0.06 & 0.02 & 0.01 & 0.01 & 0.01 & 0.01 \\
\hline $\mathrm{Na}_{2} \mathrm{O}$ & 0.02 & 0.02 & 0.60 & 0.15 & 0.31 & 0.01 & 0.04 & 0.04 & 0.43 & 0.08 & 0.03 & 0.02 \\
\hline $\mathrm{K}_{2} \mathrm{O}$ & 0.04 & 0.01 & 10.32 & 0.24 & 7.29 & 0.86 & 0.05 & 0.01 & 10.53 & 0.26 & 0.04 & 0.04 \\
\hline $\mathrm{Cr}_{2} \mathrm{O}_{3}$ & 0.09 & 0.38 & 0.07 & 0.18 & 0.00 & 0.00 & 0.02 & 0.02 & 0.28 & 0.15 & 0.68 & 0.11 \\
\hline $\mathrm{NiO}$ & 0.01 & 0.02 & 0.03 & 0.03 & 0.05 & 0.02 & 0.02 & 0.03 & 0.01 & 0.01 & 0.00 & 0.00 \\
\hline
\end{tabular}

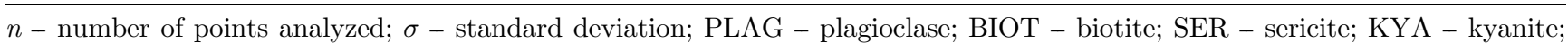
HYDMUS - hydromuscovite; PYRO - pyrophyllite; RUT - rutile.

\section{Barodia}



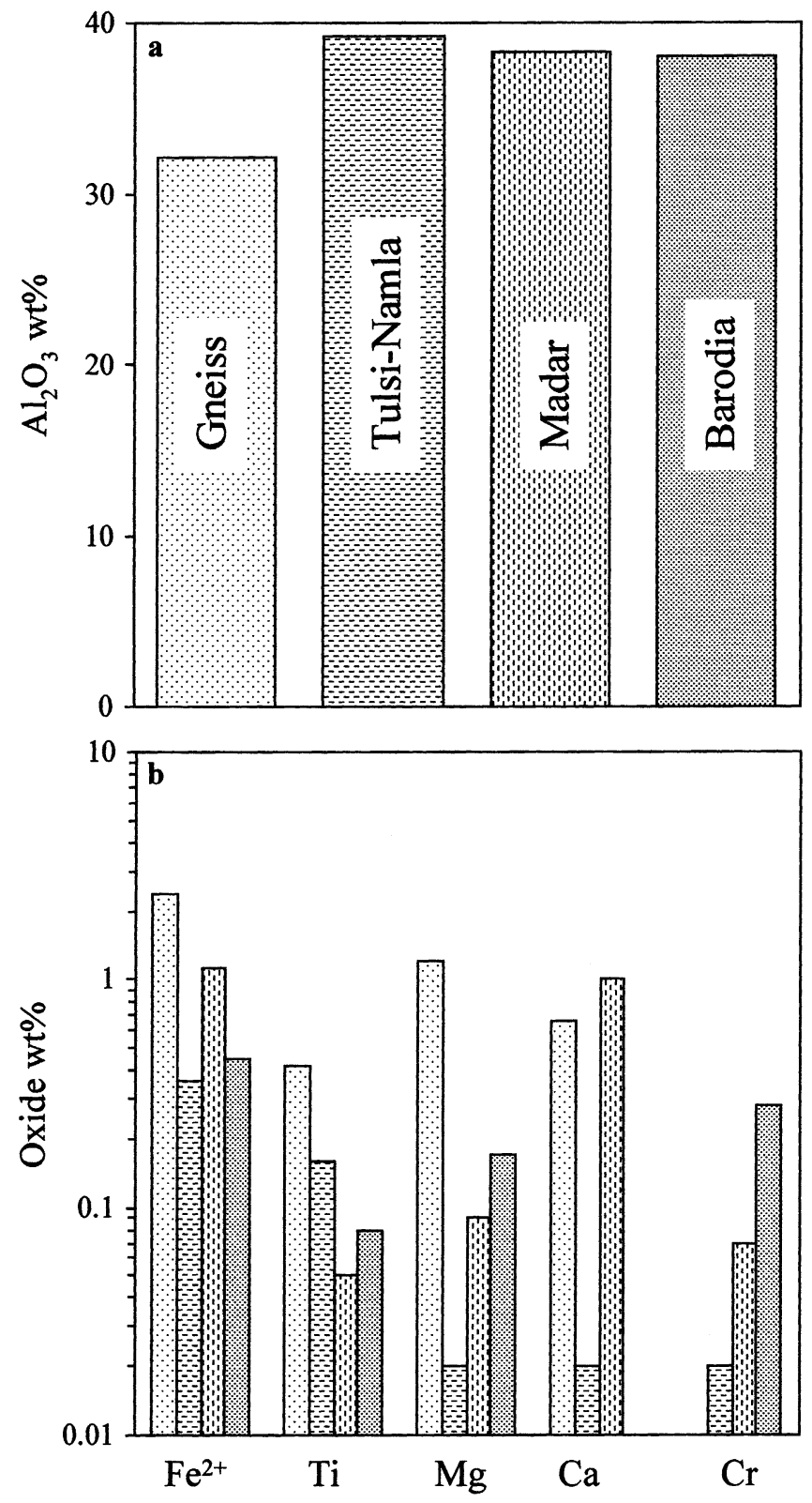

Figure 5. Differences in compositions of sericites formed due to hydrothermal alteration of plagioclase in the basement gneisses and of kyanite in the sericite deposit. (a) in $\mathrm{Al}_{2} \mathrm{O}_{3}$ content, (b) in $\mathrm{MgO}, \mathrm{Fe}_{2} \mathrm{O}_{3}, \mathrm{TiO}_{2}, \mathrm{CaO}$ and $\mathrm{Cr}_{2} \mathrm{O}_{3}$ contents.

high alumina sericites derived from break-down of kyanites are also different from low alumina sericites formed from feldspars of the basement gneisses in having lower $\mathrm{TiO}_{2}, \mathrm{FeO}$ and $\mathrm{MgO}$ (figure 5b). It has been found that all the sericites in the sericite schists are the high alumina type. The plagioclase feldspars of the basement gneiss have an anorthite content of 20 to $24 \%$. Some of the micas of the sample from Barodia quarry have slightly higher contents of $\mathrm{Cr}$.

\subsection{Major elements}

Major, trace and REE constituents of the sericite schists from Tulsi-Namla, Barodia and Madar are given in table 2. A perusal of the analyses shows that these rocks are almost wholly $(\sim 95 \%)$ made up of three constituents viz. $\mathrm{SiO}_{2}, \mathrm{Al}_{2} \mathrm{O}_{3}$ and $\mathrm{K}_{2} \mathrm{O}$ (figure 6). The silica content shows negative correlation with $\mathrm{Al}_{2} \mathrm{O}_{3}$ and $\mathrm{K}_{2} \mathrm{O}$ indicating that the sericitization of kyanites largely controls the major element composition of these rocks. Samples serially collected from the basement up to the top surface of the mica zone in Barodia quarry show much larger variations in $\mathrm{SiO}_{2}$, $\mathrm{Al}_{2} \mathrm{O}_{3}$ and $\mathrm{K}_{2} \mathrm{O}$. Silica decreases towards the top and the $\mathrm{Al}_{2} \mathrm{O}_{3}$ and $\mathrm{K}_{2} \mathrm{O}$ increase. Also depletion in $\mathrm{Fe}(\mathrm{T})$ and $\mathrm{MgO}$ is noticeable. Samples highly enriched in $\mathrm{Al}_{2} \mathrm{O}_{3}$ (c. $\left.\geq 35 \%\right)$ and $\mathrm{K}_{2} \mathrm{O}$ (c. $\geq 10 \%$ ) have been noticed from all the three areas. The compositions of these alumina- and potash-rich samples are similar to the composition of the mineral sericite, confirming the dominance of sericitic mica in the whole-rock composition.

Samples with moderate amounts of $\mathrm{Al}_{2} \mathrm{O}_{3}$ (11 to $20 \%$ ) of the Barodia and Tulsi-Namla areas show low contents of $\mathrm{K}_{2} \mathrm{O}$ (1.4 to 6 wt\%) and high amounts of silica (68 to $78 \%$ ). Even in these samples the $\mathrm{SiO}_{2}+$ $\mathrm{Al}_{2} \mathrm{O}_{3}+\mathrm{K}_{2} \mathrm{O}$ values remain close to 95 , similar to the high alumina and potash rich samples (see figure 6). All the remaining constituents are very low; the sum of the rest of the constituents $\left(\mathrm{FeO}+\mathrm{Fe}_{2} \mathrm{O}_{3}+\right.$ $\mathrm{MgO}+\mathrm{MnO}+\mathrm{CaO}+\mathrm{Na}_{2} \mathrm{O}+\mathrm{P}_{2} \mathrm{O}_{5}$ ) is generally $<2 \%$. The major element composition of sericite schists and the underlying gneisses are significantly different; the former are more enriched in $\mathrm{Al}_{2} \mathrm{O}_{3}$ and depleted in all other constituents except the $\mathrm{K}_{2} \mathrm{O}$.

$\mathrm{Al}_{2} \mathrm{O}_{3}$ and $\mathrm{TiO}_{2}$ are considered to be immobile elements during weathering, many of the Precambrian paleosols show good positive correlation between these constituents (see Sreenivas and Srinivasan 1994). The $\mathrm{Al}_{2} \mathrm{O}_{3}$ and $\mathrm{TiO}_{2}$ contents in the samples of all the three localities have been plotted in figure 7 . Serial samples of the Barodia quarry show an excellent correlation between $\mathrm{Al}_{2} \mathrm{O}_{3}$ and $\mathrm{TiO}_{2} \quad(\mathrm{r}=+0.99)$. Such a correlation is not evident from the data in the samples of the other two areas, because of cluster sampling that had to be carried out in these localities. There is a compositional gap in the range of $\mathrm{Al}_{2} \mathrm{O}_{3}$ from 20 to $35 \mathrm{wt} \%$ as in many soil profiles.

Based on a study of modern soil profiles, Maynard (1992) suggested $\mathrm{Ti} / \mathrm{Al}$ and $\mathrm{Ti} / \mathrm{Zr}$ ratios are most useful in distinguishing paleosols. According to him $\mathrm{Ti} / \mathrm{Al}$ and $\mathrm{Ti} / \mathrm{Zr}$ ratios in soils would not deviate by more than $50 \%$ from these ratios in parent rocks. The $\mathrm{Ti} / \mathrm{Al}$ ratios of the parent gneiss of Tulsi-Namla and the Ahar River Granite are 0.022 and 0.011, respectively. The average $\mathrm{Ti} / \mathrm{Al}$ values of 0.031 and 0.014 of sericite schist samples of Tulsi-Namla and Barodia respectively, are well within the limit mentioned above. The average $\mathrm{Ti} / \mathrm{Al}$ (0.004) for the samples from Madar is however, depleted when compared to the parent rock due to very low $\mathrm{Ti}$ contents. The Ti/Zr ratios of the Tulsi-Namla sericite 
Table 2. Major, trace and rare earth element analyses of sericitic rocks of Tulsi-Namla, Barodia and Madar areas.

\begin{tabular}{|c|c|c|c|c|c|c|c|}
\hline \multicolumn{8}{|c|}{ Tulsi-Namla } \\
\hline $\mathrm{Wt} \%$ & $\begin{array}{c}\text { D1S1 } \\
\text { (biotite gneiss) }\end{array}$ & D1S2A & D1S2B & $\mathrm{D} 1 \mathrm{~S} 2 \mathrm{C}$ & D1S2D & $\mathrm{D} 1 \mathrm{~S} 2 \mathrm{E}$ & D1S2F \\
\hline $\mathrm{SiO}_{2}$ & 68.80 & 74.89 & 53.17 & 45.11 & 53.46 & 48.87 & 50.05 \\
\hline $\mathrm{TiO}_{2}$ & 0.30 & 0.28 & 0.08 & 0.47 & 2.58 & 1.19 & 1.65 \\
\hline $\mathrm{Al}_{2} \mathrm{O}_{3}$ & 15.61 & 20.38 & 34.26 & 36.10 & 35.15 & 39.97 & 39.03 \\
\hline $\mathrm{Fe}_{2} \mathrm{O}_{3}$ & 0.73 & 0.00 & 0.00 & 3.53 & 0.00 & 0.00 & 0.00 \\
\hline $\mathrm{FeO}$ & 2.52 & 0.28 & 0.28 & 0.00 & 0.32 & 0.28 & 0.24 \\
\hline $\mathrm{MgO}$ & 1.31 & 0.03 & 0.05 & 0.21 & 0.03 & 0.02 & 0.13 \\
\hline $\mathrm{CaO}$ & 2.82 & 0.07 & 0.01 & 0.14 & 0.00 & 0.03 & 0.01 \\
\hline $\mathrm{Na}_{2} \mathrm{O}$ & 4.56 & 0.18 & 0.74 & 0.81 & 2.25 & 0.47 & 1.37 \\
\hline $\mathrm{K}_{2} \mathrm{O}$ & 1.24 & 1.44 & 8.91 & 0.88 & 4.67 & 5.89 & 5.24 \\
\hline $\mathrm{MnO}$ & 0.07 & 0.00 & 0.00 & 0.00 & 0.01 & 0.02 & 0.00 \\
\hline $\mathrm{P}_{2} \mathrm{O}_{5}$ & 0.11 & 0.01 & 0.02 & 0.02 & 0.12 & 0.06 & 0.01 \\
\hline LOI & 0.71 & 1.22 & 2.53 & 2.33 & 1.51 & 2.38 & 1.26 \\
\hline TOTAL & 98.78 & 98.78 & 100.05 & 99.60 & 100.10 & 99.18 & 98.99 \\
\hline \multicolumn{8}{|c|}{ Trace elements (ppm) } \\
\hline $\mathrm{Sc}$ & 2 & 15 & 23 & 13 & 42 & 12 & 22 \\
\hline $\mathrm{V}$ & 41 & 30 & 57 & 81 & 122 & 20 & 36 \\
\hline $\mathrm{Cr}$ & 8 & 230 & 184 & 113 & 54 & 6 & 62 \\
\hline $\mathrm{Ni}$ & 15 & 8 & 10 & 13 & 115 & 11 & 48 \\
\hline $\mathrm{Cu}$ & 8 & 12 & 6 & 25 & 57 & 6 & 68 \\
\hline $\mathrm{Zn}$ & 34 & 11 & 10 & 14 & 38 & 10 & 34 \\
\hline $\mathrm{Ga}$ & 6 & 17 & 54 & 29 & 35 & 28 & 46 \\
\hline $\mathrm{Rb}$ & 44 & 50 & 260 & 329 & 132 & 114 & 149 \\
\hline $\mathrm{Sr}$ & 412 & 10 & 36 & 79 & 59 & 43 & 56 \\
\hline $\mathrm{Y}$ & 13 & 1 & 3 & 5 & 4 & 19 & 14 \\
\hline $\mathrm{Zr}$ & 144 & 48 & 52 & 190 & 56 & 1557 & 988 \\
\hline $\mathrm{Nb}$ & 5 & 2 & 4 & 9 & 0.8 & 10 & 7 \\
\hline $\mathrm{Ba}$ & 492 & 16 & 33 & 159 & 57 & 27 & 42 \\
\hline Hf & 11 & 6 & 5 & 25 & 8 & 184 & 76 \\
\hline $\mathrm{Ta}$ & 0.9 & 0.5 & 0.9 & 0.6 & 0.1 & 0.8 & 0.1 \\
\hline $\mathrm{Pb}$ & 19 & 7 & 9 & 17 & 20 & 12 & 17 \\
\hline $\mathrm{Th}$ & 19 & 7 & 5 & 8 & 1 & 61 & 33 \\
\hline $\mathrm{U}$ & 0.5 & 0.4 & 0.9 & 4 & 2.5 & 5 & 2.6 \\
\hline \multicolumn{8}{|c|}{ Rare earth elements (ppm) } \\
\hline $\mathrm{La}$ & 61 & 15 & 28 & 53 & 58 & 129 & 77 \\
\hline $\mathrm{Ce}$ & 81 & 24 & 51 & 29 & 81 & 215 & 133 \\
\hline $\operatorname{Pr}$ & 11 & 4 & 9 & 4 & 17 & 32 & 21 \\
\hline $\mathrm{Nd}$ & 38 & 11 & 35 & 10 & 67 & 89 & 57 \\
\hline $\mathrm{Sm}$ & 5.5 & 1.1 & 5.4 & 2.9 & 13 & 8 & 4.1 \\
\hline $\mathrm{Eu}$ & 0.9 & 0.1 & 0.6 & 0.8 & 1.9 & 1.3 & 0.4 \\
\hline $\mathrm{Gd}$ & 3.6 & 0.6 & 1.6 & 1.9 & 5.1 & 5.4 & 3.1 \\
\hline Dy & 2.2 & 0.4 & 0.8 & 1 & 1.5 & 3.2 & 2 \\
\hline Er & 0.7 & 0.2 & 0.3 & 0.3 & 0.4 & 1.7 & 1 \\
\hline $\mathrm{Yb}$ & 0.9 & 0.4 & 0.5 & 1.1 & 0.7 & 4.4 & 2.3 \\
\hline $\mathrm{Lu}$ & 0.1 & 0.04 & 0.05 & 0.1 & 0.1 & 0.4 & 0.3 \\
\hline$\Sigma \mathrm{REE}$ & 206 & 57 & 133 & 105 & 247 & 491 & 302 \\
\hline
\end{tabular}

Serially collected samples from Barodia

\begin{tabular}{|c|c|c|c|c|c|c|c|}
\hline \multirow[b]{2}{*}{$\mathrm{Wt} \%$} & \multicolumn{6}{|c|}{ Towards upper contact } & \multirow[b]{2}{*}{$\mathrm{W} 4 / 6$} \\
\hline & $\mathrm{B} 4 / 6$ & $\mathrm{C} 4 / 6$ & $\mathrm{~F} 4 / 6$ & $\mathrm{G} 4 / 6$ & $\mathrm{P} 4 / 6$ & $\mathrm{R} 4 / 6$ & \\
\hline $\mathrm{SiO}_{2}$ & 79.54 & 78.26 & 77.83 & 74.57 & 70.66 & 68.18 & 45.62 \\
\hline $\mathrm{TiO}_{2}$ & 0.02 & 0.14 & 0.12 & 0.22 & 0.27 & 0.36 & 1.31 \\
\hline $\mathrm{Al}_{2} \mathrm{O}_{3}$ & 12.99 & 14.28 & 14.86 & 17.05 & 19.21 & 20.86 & 37.07 \\
\hline $\mathrm{Fe}_{2} \mathrm{O}_{3}$ & 0.18 & 0.29 & 0.53 & 0.29 & 0.43 & 0.32 & 0.09 \\
\hline $\mathrm{FeO}$ & 0.28 & 0.32 & 0.28 & 0.28 & 0.20 & 0.36 & 0.28 \\
\hline $\mathrm{MnO}$ & 0.00 & 0.00 & 0.00 & 0.00 & 0.00 & 0.01 & 0.00 \\
\hline $\mathrm{MgO}$ & 0.61 & 0.69 & 0.45 & 0.77 & 0.16 & 0.71 & 0.28 \\
\hline $\mathrm{CaO}$ & 0.06 & 0.04 & 0.01 & 0.02 & 0.01 & 0.03 & 0.03 \\
\hline $\mathrm{Na}_{2} \mathrm{O}$ & 0.11 & 0.12 & 0.19 & 0.14 & 0.14 & 0.24 & 0.00 \\
\hline $\mathrm{K}_{2} \mathrm{O}$ & 4.20 & 4.35 & 5.08 & 5.32 & 6.17 & 6.88 & 10.85 \\
\hline
\end{tabular}


Table 2. (Continued)

\begin{tabular}{|c|c|c|c|c|c|c|c|c|c|c|c|}
\hline \multicolumn{12}{|c|}{ Serially collected samples from Barodia } \\
\hline \multicolumn{12}{|c|}{ Towards upper contact } \\
\hline $\mathrm{Wt} \%$ & \multicolumn{2}{|c|}{$\mathrm{B} 4 / 6$} & $\mathrm{C} 4 / 6$ & \multicolumn{2}{|c|}{$\mathrm{F} 4 / 6$} & \multicolumn{2}{|l|}{ G4/6 } & $\mathrm{P} 4 / 6$ & \multicolumn{2}{|l|}{$\mathrm{R} 4 / 6$} & $\mathrm{~W} 4 / 6$ \\
\hline $\mathrm{P}_{2} \mathrm{O}_{5}$ & \multicolumn{2}{|c|}{0.01} & 0.00 & \multicolumn{2}{|c|}{0.00} & 0.00 & & 0.01 & 0.02 & & 0.01 \\
\hline LOI & & & 0.58 & & & 0.70 & & 0.88 & 1.31 & & 3.52 \\
\hline Total & & & 99.07 & 100 & & 99.36 & & 98.14 & 99.28 & & 99.06 \\
\hline Trace ele & ents $(\mathrm{pl}$ & & & & & & & & & & \\
\hline $\mathrm{Sc}$ & & & 4 & 6 & & 5 & & 8 & 7 & & 19 \\
\hline $\mathrm{V}$ & 1 & & 17 & 19 & & 10 & & 25 & 22 & & 316 \\
\hline $\mathrm{Cr}$ & & & 10 & 24 & & 4 & & 25 & 16 & & 895 \\
\hline $\mathrm{Ni}$ & & & 7 & 9 & & 7 & & 9 & 8 & & 11 \\
\hline $\mathrm{Cu}$ & & & 5 & 6 & & 4 & & 5 & 5 & & 7 \\
\hline $\mathrm{Zn}$ & 1 & & 5 & 6 & & 6 & & 8 & 10 & & 11 \\
\hline $\mathrm{Ga}$ & & & 20 & 24 & & 20 & & 27 & 27 & & 59 \\
\hline $\mathrm{Rb}$ & 11 & & 121 & 145 & & 131 & & 146 & 156 & & 284 \\
\hline $\mathrm{Sr}$ & & & 8 & 11 & & 7 & & 8 & 8 & & 18 \\
\hline $\mathrm{Y}$ & & & 6 & 14 & & 17 & & 9 & 8 & & 5 \\
\hline $\mathrm{Zr}$ & 9 & & 206 & 551 & & 640 & & 394 & 322 & & 354 \\
\hline $\mathrm{Nb}$ & & & 6 & 8 & & 15 & & 6 & 6 & & 3 \\
\hline $\mathrm{Ba}$ & 17 & & 267 & 259 & & 370 & & 248 & 327 & & 163 \\
\hline Hf & 1 & & 33 & 78 & & 112 & & 51 & 41 & & 35 \\
\hline $\mathrm{Ta}$ & & & 0.6 & 0 & & 1.2 & & 0.5 & 0.5 & & 0.2 \\
\hline $\mathrm{Pb}$ & & & 6 & 6 & & 5 & & 5 & 6 & & 7 \\
\hline $\mathrm{Th}$ & & & 3 & 17 & & 31 & & 10 & 17 & & 19 \\
\hline $\mathrm{U}$ & & & 3 & 2 & & 8 & & 3 & 4 & & 0.8 \\
\hline Rare ear & elemen & pm) & & & & & & & & & \\
\hline $\mathrm{La}$ & & & 9 & 6 & & 56 & & 16 & 38 & & 8 \\
\hline $\mathrm{Ce}$ & & & 11 & 5 & & 72 & & 20 & 51 & & 11 \\
\hline $\operatorname{Pr}$ & & & 2 & 1 & & 10 & & 3 & 7 & & 3 \\
\hline $\mathrm{Nd}$ & & & 4 & 4 & & 26 & & 8 & 19 & & 8 \\
\hline $\mathrm{Sm}$ & & & 1.5 & & & 3 & & 1 & 1.6 & & 2 \\
\hline $\mathrm{Eu}$ & & & 0.3 & 0 & & 0.2 & & 0.5 & 0.5 & & 0.3 \\
\hline $\mathrm{Gd}$ & & & 0.7 & 3 & & 1 & & 0.8 & 1.5 & & 1.1 \\
\hline Dy & & & 0.8 & 2 & & 1.3 & & 1.2 & 1 & & 0.8 \\
\hline $\mathrm{Er}$ & & & 0.6 & 1 & & 0.8 & & 0.6 & 0.7 & & 0.5 \\
\hline $\mathrm{Yb}$ & & & 1.5 & 3 & & 2.5 & & 1.9 & 2 & & 0.6 \\
\hline $\mathrm{Lu}$ & & & 0.1 & & & 0.3 & & 0.3 & 0.2 & & 0.1 \\
\hline$\Sigma \mathrm{REE}$ & & & 32 & 24 & & 180 & & 54 & 124 & & 36 \\
\hline & & & & & & adar & & & & & \\
\hline $\mathrm{Wt} \%$ & $\mathrm{C} 4 / 1$ & $\mathrm{D} 4 / 1$ & $\mathrm{E} 4 / 1$ & $\mathrm{~F} 4 / 1$ & $\mathrm{G} 4 / 1$ & $\mathrm{H} 4 / 1$ & $\mathrm{~J} 4 / 1$ & $\mathrm{~K} 4 / 1$ & $\mathrm{~L} 4 / 1$ & $\mathrm{M} 4 / 1$ & $\mathrm{O} 4 / 1$ \\
\hline $\mathrm{SiO}_{2}$ & 52.69 & 49.05 & 46.88 & 48.33 & 47.51 & 52.18 & 48.91 & 47.75 & 50.27 & 49.15 & 49.49 \\
\hline $\mathrm{TiO}_{2}$ & 0.29 & 0.23 & 0.07 & 0.13 & 0.12 & 0.07 & 0.14 & 0.01 & 0.05 & 0.27 & 0.03 \\
\hline $\mathrm{Al}_{2} \mathrm{O}_{3}$ & 36.97 & 35.19 & 38.94 & 36.54 & 42.42 & 34.52 & 36.39 & 37.75 & 36.01 & 36.97 & 36.18 \\
\hline $\mathrm{Fe}_{2} \mathrm{O}_{3}$ & 0.16 & 1.44 & 0.55 & 0.41 & 0.37 & 1.78 & 0.50 & 0.64 & 0.79 & 0.44 & 0.49 \\
\hline $\mathrm{FeO}$ & 0.24 & 0.24 & 0.28 & 0.28 & 0.24 & 0.28 & 0.24 & 0.24 & 0.28 & 0.24 & 0.24 \\
\hline $\mathrm{MnO}$ & 0.00 & 0.00 & 0.00 & 0.00 & 0.00 & 0.00 & 0.00 & 0.00 & 0.00 & 0.00 & 0.00 \\
\hline $\mathrm{MgO}$ & 0.05 & 0.16 & 0.12 & 0.08 & 0.19 & 0.29 & 0.05 & 0.09 & 0.14 & 0.06 & 0.13 \\
\hline $\mathrm{CaO}$ & 0.01 & 0.02 & 0.02 & 0.02 & 0.04 & 0.03 & 0.01 & 0.03 & 0.03 & 0.01 & 0.03 \\
\hline $\mathrm{Na}_{2} \mathrm{O}$ & 0.28 & 0.41 & 0.51 & 0.44 & 0.30 & 0.39 & 0.48 & 0.48 & 0.44 & 0.52 & 0.35 \\
\hline $\mathrm{K}_{2} \mathrm{O}$ & 4.20 & 10.15 & 10.44 & 10.08 & 5.85 & 8.78 & 10.18 & 10.46 & 9.95 & 9.87 & 10.25 \\
\hline $\mathrm{P}_{2} \mathrm{O}_{5}$ & 0.06 & 0.04 & 0.02 & 0.02 & 0.06 & 0.05 & 0.02 & 0.01 & 0.02 & 0.02 & 0.05 \\
\hline LOI & 4.28 & 1.40 & 3.67 & 2.44 & 3.15 & 2.77 & 2.64 & 1.50 & 2.97 & 1.98 & 1.47 \\
\hline TOTAL & 99.23 & 98.33 & 101.50 & 98.77 & 100.25 & 101.14 & 99.56 & 98.96 & 101.95 & 100.53 & 98.71 \\
\hline Trace ele & ents ( $\mathrm{pl}$ & & & & & & & & & & \\
\hline $\mathrm{Sc}$ & 6 & 6 & 7 & 9 & 8 & 7 & 9 & 10 & 8 & 12 & 12 \\
\hline $\mathrm{V}$ & 38 & 46 & 47 & 51 & 43 & 252 & 77 & 109 & 58 & 61 & 62 \\
\hline $\mathrm{Cr}$ & 20 & 29 & 18 & 51 & 17 & 34 & 32 & 19 & 13 & 23 & 120 \\
\hline $\mathrm{Ni}$ & 6 & 12 & 5 & 5 & 8 & 13 & 6 & 6 & 9 & 7 & 6 \\
\hline $\mathrm{Cu}$ & 99 & 27 & 2 & 4 & 161 & 133 & 6 & 4 & 2 & 4 & 3 \\
\hline $\mathrm{Zn}$ & 30 & 21 & 10 & 10 & 27 & 33 & 15 & 12 & 9 & 17 & 10 \\
\hline
\end{tabular}


Table 2. (Continued)

\begin{tabular}{|c|c|c|c|c|c|c|c|c|c|c|c|}
\hline \multicolumn{12}{|c|}{ Madar } \\
\hline $\mathrm{Wt} \%$ & $\mathrm{C} 4 / 1$ & $\mathrm{D} 4 / 1$ & $\mathrm{E} 4 / 1$ & $\mathrm{~F} 4 / 1$ & $\mathrm{G} 4 / 1$ & $\mathrm{H} 4 / 1$ & $\mathrm{~J} 4 / 1$ & $\mathrm{~K} 4 / 1$ & $\mathrm{~L} 4 / 1$ & M4/1 & $\mathrm{O} 4 / 1$ \\
\hline $\mathrm{Ga}$ & 31 & 33 & 40 & 49 & 41 & 33 & 41 & 53 & 39 & 53 & 46 \\
\hline $\mathrm{Rb}$ & 95 & 247 & 263 & 299 & 143 & 241 & 285 & 291 & 251 & 289 & 311 \\
\hline $\mathrm{Sr}$ & 81 & 72 & 59 & 64 & 63 & 75 & 66 & 42 & 50 & 60 & 70 \\
\hline $\mathrm{Y}$ & 6 & 7 & 5 & 9 & 46 & 6 & 7 & 4 & 5 & 5 & 11 \\
\hline $\mathrm{Zr}$ & 125 & 195 & 103 & 154 & 258 & 102 & 208 & 86 & 102 & 125 & 280 \\
\hline $\mathrm{Nb}$ & 4 & 3 & 1 & 2 & 4 & 4 & 2 & 2 & 1 & 1 & 4 \\
\hline $\mathrm{Ba}$ & 77 & 124 & 111 & 117 & 94 & 111 & 125 & 120 & 107 & 113 & 113 \\
\hline Hf & 3 & 5 & 3 & 4 & 6 & 2 & 5 & 2 & 2 & 3 & 6 \\
\hline $\mathrm{Ta}$ & 0.2 & 0.2 & 0.2 & 0.1 & 0.1 & 0.1 & 0.1 & 0.2 & 0.1 & 0.1 & 0.1 \\
\hline $\mathrm{Pb}$ & 16 & 13 & 9 & 11 & 10 & 11 & 12 & 8 & 9 & 9 & 10 \\
\hline $\mathrm{Th}$ & 28 & 21 & 15 & 15 & 10 & 10 & 16 & 4 & 8 & 5 & 22 \\
\hline $\mathrm{U}$ & 2 & 4 & 2 & 2 & 2 & 2 & 2 & 1 & 2 & 2 & 3 \\
\hline \multicolumn{12}{|c|}{ Rare earth elements (ppm) } \\
\hline $\mathrm{La}$ & 38 & 22 & 23 & 18 & 11 & 17 & 22 & 4 & 10 & 5 & 31 \\
\hline $\mathrm{Ce}$ & 67 & 41 & 43 & 33 & 18 & 31 & 45 & 9 & 22 & 11 & 57 \\
\hline $\operatorname{Pr}$ & 8 & 5 & 5 & 4 & 2 & 4 & 6 & 1 & 3 & 2 & 7 \\
\hline $\mathrm{Nd}$ & 24 & 22 & 19 & 12 & 6 & 17 & 20 & 5 & 9 & 6 & 23 \\
\hline $\mathrm{Sm}$ & 4 & 4 & 2 & 2 & 2 & 3 & 2 & 1 & 1 & 1 & 3 \\
\hline $\mathrm{Eu}$ & 0.6 & 0.7 & 0.4 & 0.4 & 0.3 & 0.6 & 0.5 & 0.2 & 0.3 & 0.2 & 0.7 \\
\hline $\mathrm{Gd}$ & 2 & 2 & 1.5 & 1.3 & 1.4 & 1.7 & 1.9 & 0.3 & 0.9 & 0.8 & 2 \\
\hline Dy & 0.9 & 1 & 0.9 & 1.1 & 1.6 & 0.7 & 1.0 & 0.5 & 0.7 & 0.6 & 1.1 \\
\hline Er & 0.7 & 0.8 & 0.7 & 0.7 & 1.2 & 0.6 & 0.7 & 0.4 & 0.4 & 0.6 & 0.9 \\
\hline $\mathrm{Yb}$ & 1.5 & 1.6 & 0.9 & 1.3 & 2 & 1.1 & 1.5 & 0.8 & 0.8 & 1.1 & 1.5 \\
\hline $\mathrm{Lu}$ & 0.1 & 0.2 & 0.2 & 0.2 & 0.3 & 0.1 & 0.2 & 0.1 & 0.1 & 0.1 & 0.3 \\
\hline$\Sigma \mathrm{REE}$ & 148 & 101 & 97 & 75 & 47 & 77 & 101 & 23 & 49 & 29 & 128 \\
\hline
\end{tabular}

schist samples (average $=14.35$, except sample \# $\mathrm{D} 1 \mathrm{~S} 2 \mathrm{D})$ is very close to the value of the parent gneiss (12.5).

\subsubsection{Weathering indices}

Ideally the degree of chemical weathering in a soil or paleosol should be calculated using gains and losses of material from a constant volume (Retallack et al
1984). However, this is not feasible in the case of buried and metamorphosed soil profiles, where compaction exceeds any correction limit. In such cases chemical weathering can be estimated by normalizing the analyses to a chemical component such as $\mathrm{Al}_{2} \mathrm{O}_{3}$, $\mathrm{TiO}_{2}, \mathrm{ZrO}_{2}$ or $\mathrm{Fe}_{2} \mathrm{O}_{3}$ which is assumed to be retained during chemical weathering (Retallack et al 1984). Of the several indices proposed, the Chemical Index of Alteration (CIA) proposed by Nesbitt and Young

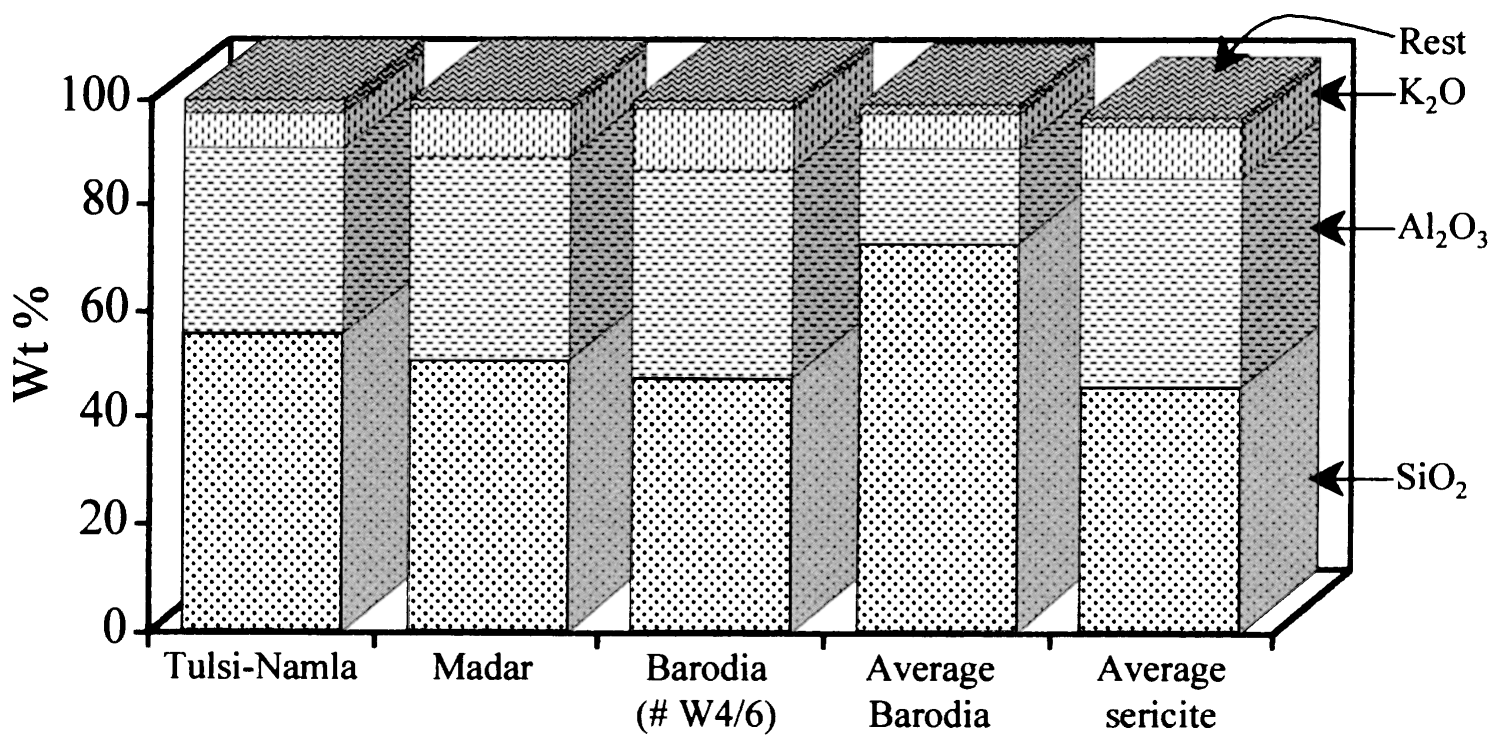

Figure 6. Major element compositions of sericite schists depicting that they are wholly made up of three constituents, viz. $\mathrm{SiO}_{2}$, $\mathrm{Al}_{2} \mathrm{O}_{3}, \mathrm{~K}_{2} \mathrm{O}$. 


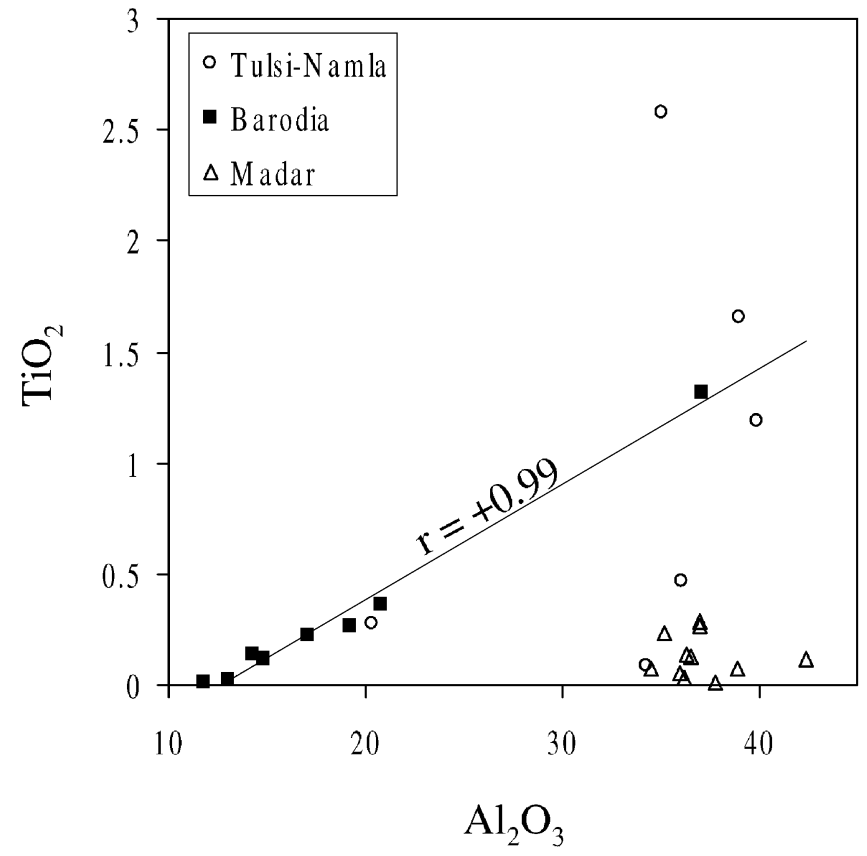

Figure 7. $\mathrm{Al}_{2} \mathrm{O}_{3}$ vs. $\mathrm{TiO}_{2}$ diagram for sericite schists at the MGC - Aravalli contact. Note the good correlation in serially collected samples of Barodia quarry.
(1982) and the Chemical Index of Weathering (CIW) proposed by Harnois (1988) have been used extensively for estimating the intensity of weathering. The CIA is the ratio of molar proportion of alumina and alkalis $\left[100\left(\mathrm{Al}_{2} \mathrm{O}_{3}\right) /\left(\mathrm{Al}_{2} \mathrm{O}_{3}+\right.\right.$ silicate $\mathrm{CaO}+$ $\left.\mathrm{Na}_{2} \mathrm{O}+\mathrm{K}_{2} \mathrm{O}\right)$ ]. CIW is the CIA calculated on $\mathrm{K}_{2} \mathrm{O}$ free basis. In a recent work Fedo et al (1995) have shown that CIW values can be misleading in deciphering the intensity of weathering, since they do not account for the alumina content associated with potash feldspar, which constitutes a rock forming mineral in the upper crust. Instead of CIW, Fedo et al (1995) therefore have proposed Plagioclase Index of Alteration (PIA) which is $100\left(\mathrm{Al}_{2} \mathrm{O}_{3}-\mathrm{K}_{2} \mathrm{O}\right) /\left(\mathrm{Al}_{2} \mathrm{O}_{3}\right.$ + silicate $\left.\mathrm{CaO}+\mathrm{Na}_{2} \mathrm{O}-\mathrm{K}_{2} \mathrm{O}\right)$. The PIA is very useful where plagioclase alteration needs to be estimated. Further, these authors have suggested a method to correct for the metasomatically introduced $\mathrm{K}_{2} \mathrm{O}$ in the paleosols or pelites by using the predicted weathering trends of parent rocks plotted in the $\mathrm{Al}_{2} \mathrm{O}_{3}-\left(\mathrm{CaO}+\mathrm{Na}_{2} \mathrm{O}\right)-\mathrm{K}_{2} \mathrm{O}(\mathrm{A}-\mathrm{CN}-\mathrm{K}$, all in mole proportions) diagram.

The CIA, CIW and PIA values of sericite rock samples are given in table 3. The CIA values for sericite schists overlying the biotite gneisses from the Tulsi-Namla area show a range between 73 and 91 .

Those which overlie the Ahar River granite from

Table 3. CIA, pre-metasomatic CIA, CIW and PIA values along with the percentage of increase in the CIA values after correction for the metasomatically added $\mathrm{K}_{2} \mathrm{O}$ for the sericitic schists.

\begin{tabular}{|c|c|c|c|c|c|}
\hline Sample & CIA & $\begin{array}{l}\text { Pre-metasomatic } \\
\text { CIA values }\end{array}$ & CIW & PIA & $\begin{array}{c}\text { Increase in the CIA values } \\
\text { when pre-metasomatic CIA } \\
\text { values are calculated }\end{array}$ \\
\hline \multicolumn{6}{|l|}{ Madar } \\
\hline $\mathrm{C} 4 / 1$ & 88.3 & 88.3 to 100 & 99.1 & 99.0 & \\
\hline D4/1 & 75.2 & 83.0 & 98.3 & 97.5 & 10.4 \\
\hline $\mathrm{E} 4 / 1$ & 76.3 & 81.0 & 97.9 & 97.1 & 6.2 \\
\hline $\mathrm{F} 4 / 1$ & 75.9 & 81.0 & 98.1 & 97.3 & 6.8 \\
\hline $\mathrm{G} 4 / 1$ & 86.3 & 86.3 to 100 & 99.0 & 98.8 & \\
\hline $\mathrm{H} 4 / 1$ & 77.4 & 81.0 & 98.4 & 97.8 & 4.6 \\
\hline $\mathrm{J} 4 / 1$ & 75.6 & 83.0 & 98.0 & 97.1 & 9.9 \\
\hline $\mathrm{K} 4 / 1$ & 75.7 & 83.0 & 97.9 & 97.0 & 9.7 \\
\hline $\mathrm{L} 4 / 1$ & 75.8 & 83.0 & 98.0 & 97.2 & 9.5 \\
\hline M4/1 & 76.3 & 83.0 & 97.8 & 97.0 & 8.8 \\
\hline $\mathrm{O} 4 / 1$ & 75.7 & 81.0 & 98.6 & 98.0 & 7.0 \\
\hline \multicolumn{6}{|c|}{ Barodia quarry } \\
\hline $\mathrm{B} 4 / 6$ & 73.0 & 83.0 & 98.2 & 97.2 & 14.2 \\
\hline $\mathrm{C} 4 / 6$ & 74.1 & 83.0 & 98.4 & 97.7 & 12.3 \\
\hline $\mathrm{F} 4 / 6$ & 71.8 & 83.0 & 98.2 & 97.3 & 11.9 \\
\hline $\mathrm{G} 4 / 6$ & 73.9 & 81.0 & 97.8 & 96.5 & 12.8 \\
\hline $\mathrm{P} 4 / 6$ & 73.6 & 83.0 & 98.8 & 98.1 & 12.9 \\
\hline $\mathrm{R} 4 / 6$ & 72.7 & 81.0 & 98.0 & 97.0 & 11.0 \\
\hline $\mathrm{W} 4 / 6$ & 75.9 & 85 to 100 & 99.9 & 99.8 & \\
\hline \multicolumn{6}{|c|}{ Tulsi-Namla } \\
\hline D1S2A & 91.2 & & 98.1 & 97.9 & \\
\hline D1S2B & 76.0 & 87.5 & 96.7 & 95.4 & 15.2 \\
\hline D1S2C & 73.1 & 87.5 & 95.9 & 94.1 & 19.8 \\
\hline D1S2D & 80.6 & 83.0 & 91.1 & 89.8 & 3.0 \\
\hline D1S2E & 85.0 & 90.0 & 98.3 & 98.0 & 5.9 \\
\hline D1S2F & 83.1 & 86.2 & 94.6 & 93.7 & 3.7 \\
\hline
\end{tabular}


Barodia and Madar areas exhibit a narrower and lower range of CIA ( 72 to 76,75 to 78 ; respectively). The CIW values of the samples from Barodia quarry and Madar areas are close to 100, those of Tulsi-Namla are slightly less (91 to 98). The PIA values of samples from all the three areas are slightly less than their CIW values and are always higher than 90 .

\subsubsection{Pre-metasomatic CIA values (correction for metasomatically introduced $\mathrm{K}_{2} \mathrm{O}$ )}

Fedo et al (1995) have shown that the metasomatically introduced potash content will affect the primary compositions of shales or paleosols, so that their plot which should normally be along the predicted weathering trends of their parent igneous rocks, shifts towards the $\mathrm{K}_{2} \mathrm{O}$ apex in the $\mathrm{A}-\mathrm{CN}-\mathrm{K}$ space. The average compositions of the biotite gneisses, and the Ahar River granite along with the bulk compositions of the sericite schists have been plotted on the A-CN-K diagram (figure 8a,b). Most of the sericite schist samples plot well removed from the predicted weathering trends of gneisses and granites. The compositions of samples are shifted towards $\mathrm{K}$ along A-K join. The pre-metasomatic CIA values for these samples have been calculated following the method suggested by Fedo et al (1995) and are given in table 3. It can be observed that the pre-metasomatic CIA values of the sericite schist samples of the Tulsi-Namla area are $>83$ and reach a maximum of 90 , while those of Barodia and Madar range between 85 and 100 . The broader range observed for the samples of Tulsi-Namla indicate variable degrees of metasomatism yielding a metasomatic trend (see figure 8a). These characteristics corroborate the petrographic observations of the sericite schists discussed earlier, which shows more relicts of kyanites in the rock from Tulsi-Namla area. The pre-metasomatic CIA values are very similar to the values of residual clays given by Nesbitt and Young (1982). The samples close to the basement Ahar River Granite from the Barodia quarry exhibit a restricted range of pre-metasomatic CIA values between 81 and 84 which are similar to the CIA value of illite. These illite-like CIA values coupled with moderate alumina contents in the chloritequartz-sericite samples of Barodia indicate that they may represent either moderately or incipiently weathered products of the Ahar River Granite.

\subsubsection{Iron contents in sericite schists}

Since a paleosol nature of protoliths of sericite schists is suspected, the behavior of iron in these rocks is very important to understand because it is very useful in estimating $\mathrm{PO}_{2}$ of the Precambrian atmosphere. The total iron as $\mathrm{FeO}$ in samples of all the three areas is less when compared to the parent gneiss and granite indicating loss of iron. However, Ohmoto (1996) suggested that comparison of weight percentages of $\mathrm{FeO}$ may not be truly representative of gain or loss from a parent rock. He suggested instead that $\mathrm{Fe}^{2+} /$ $\mathrm{Ti}, \mathrm{Fe}^{3+} / \mathrm{Ti}$ and $\Sigma \mathrm{Fe} / \mathrm{Ti}$ (atomic ratios) are very useful in estimating the gains and losses because $\mathrm{Ti}$ is highly immobile. The $\Sigma \mathrm{Fe} / \mathrm{Ti}\left(\mathrm{Fe}\right.$ as $\left.\mathrm{Fe}^{2+}\right)$ ratios of gneiss and granite are 13.7 and 10, respectively. The sericite schist samples from Tulsi-Namla overlying the gneisses have $\Sigma \mathrm{Fe} / \mathrm{Ti}$ values ranging between 0.2 and 8.7 indicating a significant loss of iron. It can also be seen that except for one sample (\# D1S2C), all the samples show $\mathrm{Fe}_{2} \mathrm{O}_{3}$ contents below detection level. Samples from the Barodia and Madar areas which overlie the Ahar River Granite show a large range of $\Sigma \mathrm{Fe} / \mathrm{Ti}$ values between 0.4 and 105 and some samples have enriched values when compared to the granite. In the serial samples of Barodia, the sample near its upper contact (\# W4/6) shows the lowest $\Sigma \mathrm{Fe} / \mathrm{Ti}$ value of 0.4 and the samples collected near the lower contact show higher values. Based on the $\mathrm{Fe}^{2+} / \mathrm{Ti}$ and $\mathrm{Fe}^{3+} / \mathrm{Ti}$ relationship, Ohmoto (1996) divided Precambrian paleosols into four different types: Oxidized (O-type), Reduced (R-type), Hydrothermally altered (H-type) and Mixed process (M-type). The $\mathrm{Fe}^{2+} / \mathrm{Ti}$ versus $\mathrm{Fe}^{3+} / \mathrm{Ti}$ ratios for Barodia and Madar samples are plotted in figure 9(a,b). Tulsi-Namla samples are excluded because of the below detection level abundance of $\mathrm{Fe}^{3+}$ and $\mathrm{Fe}^{2+}$. It can be seen that the serial samples of Barodia quarry and the cluster samples of Madar show very good positive correlations as in the case of H-type paleosols. Sample \# B4/6 is from the lowest part of the sericite schist horizon among the Barodia samples; it has highest $\Sigma \mathrm{Fe} / \mathrm{Ti}$ value of 28 , indicating enrichment of iron in the lower portion.

\subsection{Trace and rare earth element (REE) composition}

In sericite schists with reference to the basement gneisses, $\mathrm{Rb}, \mathrm{Cr}$ and $\mathrm{Zr}$ are enriched, $\mathrm{Sr}$ is depleted, and elements like $\mathrm{Ni}, \mathrm{V}, \mathrm{Zn}$ and $\mathrm{Pb}$ are unaffected. Among the high field strength elements (HFSE), Zr and Hf show enrichment especially in the samples from Tulsi-Namla and Barodia, whereas $\mathrm{Y}$ and $\mathrm{Nb}$ are not very much affected. The increase in $\mathrm{Zr}$ and $\mathrm{Hf}$ can be related to increases in the abundance of zircon and rutile in the sericite schist as compared to the gneisses. $\mathrm{Zr}$, Hf, Y, Nb show good positive correlation among themselves in samples of Tulsi-Namla and Barodia corroborating the inference that the accessory heavy mineral fraction has controlled the abundance of these HFSE in these samples. $\mathrm{Al}_{2} \mathrm{O}_{3}$ content and $\mathrm{Th} / \mathrm{Sc}$ ratios in the samples of Tulsi-Namla area show moderate positive correlation with $\mathrm{Zr}, \mathrm{Hf}, \mathrm{Y}$, Nb and RREE. Although this relation generally holds, the immobility of $\mathrm{Zr}$ in weathering environments leads to deviation from this relation. For example, the 


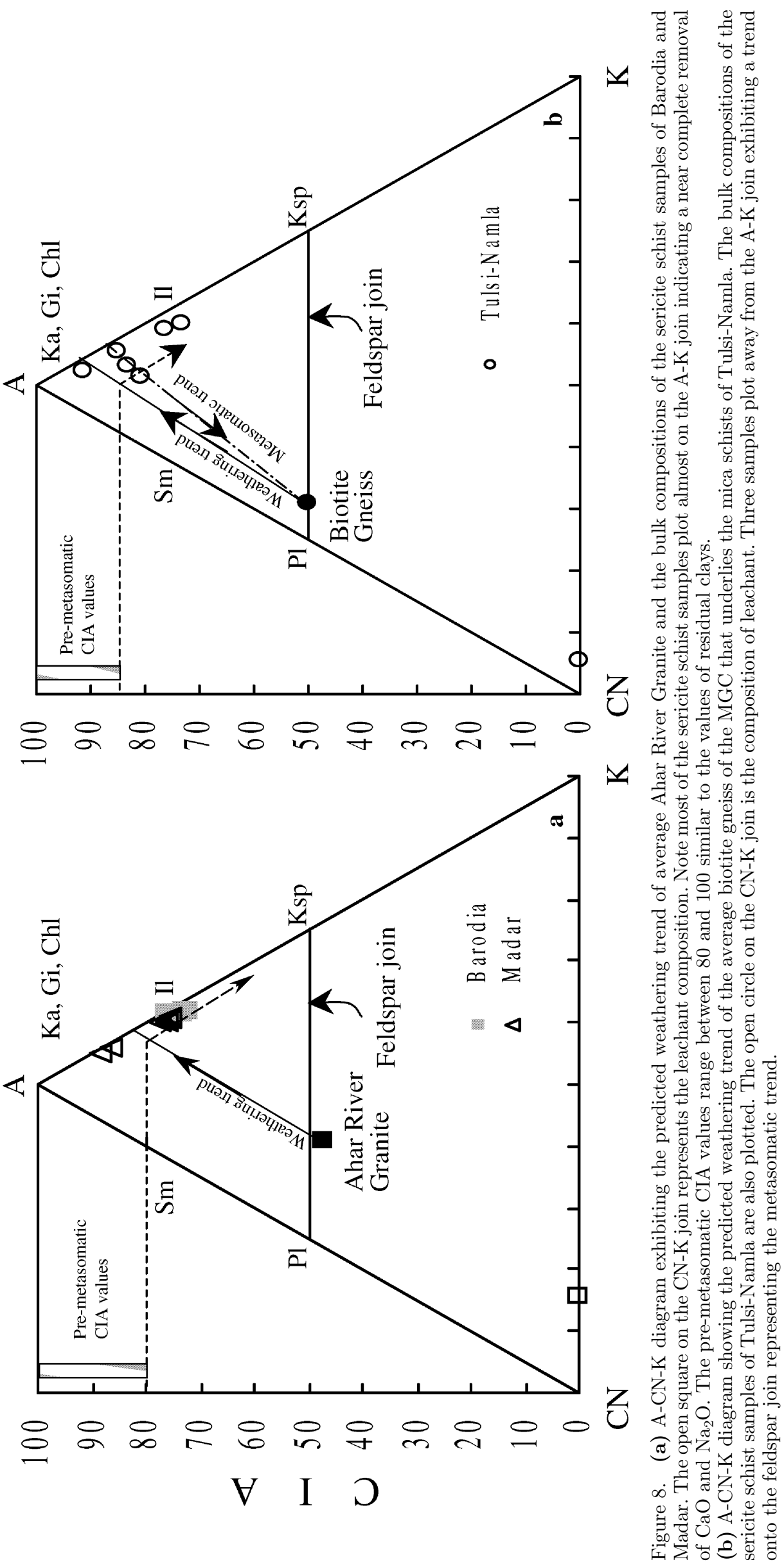



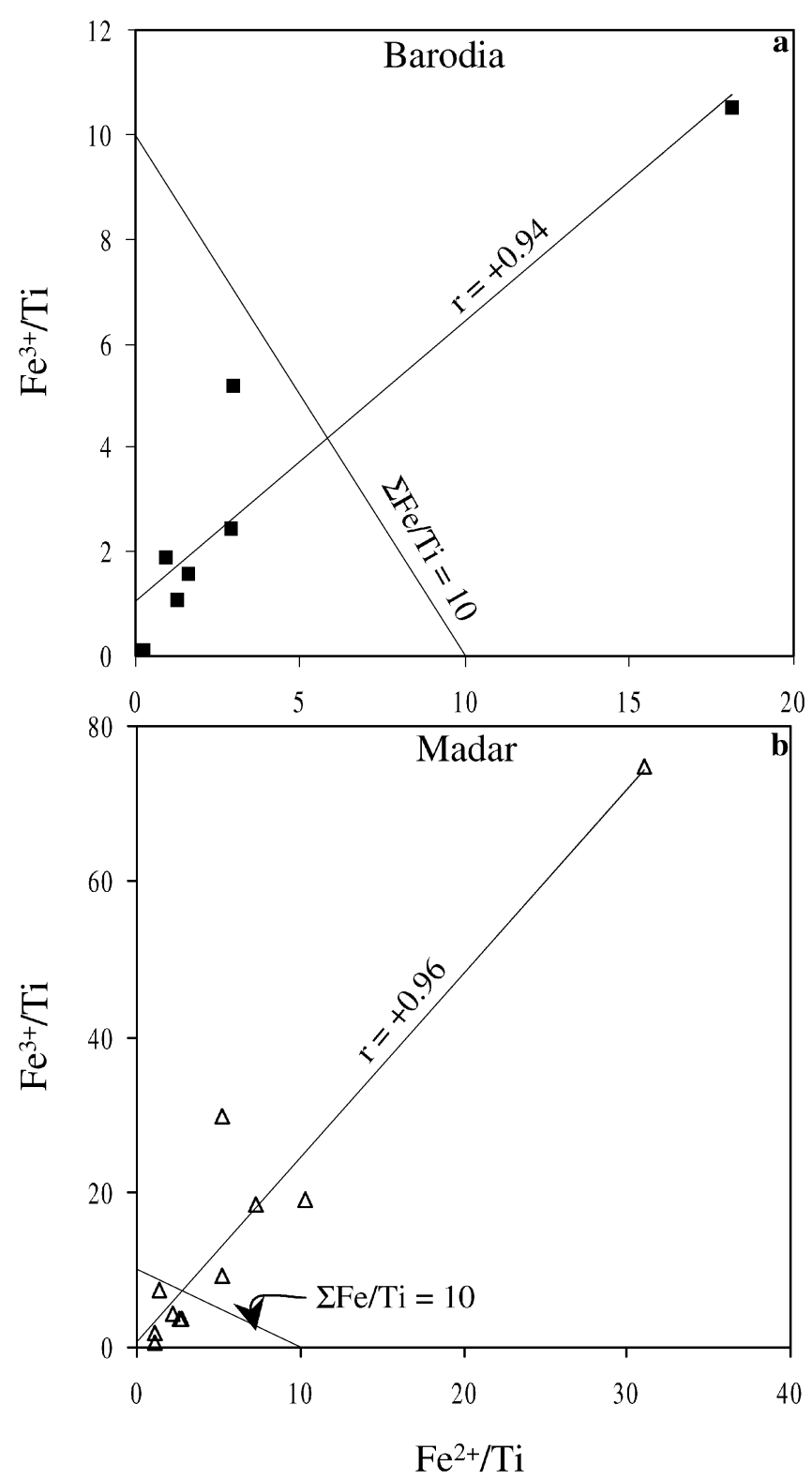

Figure 9. $\mathrm{Fe}^{2+} / \mathrm{Ti}$ vs. $\mathrm{Fe}^{3+} / \mathrm{Ti}$ plots of (a) Barodia and (b) Madar palaesols. Note good positive correlation in both the areas resembling the ' $\mathrm{H}$ type' paleosols (Ohmoto 1996). $\Sigma \mathrm{Fe} /$ $\mathrm{Ti}=10$ is the value of Ahar River Granite. It can be noticed that some samples in both the regions exhibit higher $\Sigma \mathrm{Fe} / \mathrm{Ti}$ ratios when compared to the parent rock, indicating that they may also have characteristics of ' $\mathrm{M}$ type' paleosols.

zircon-rich layers of gneissic bands retain Zr-rich character in a weathering profile, although they may not be the layers richest in $\mathrm{Al}_{2} \mathrm{O}_{3}$ as for example in some layers in lower parts of the soil horizon. The Th/ Sc values are not similar for samples from Barodia, Madar and Tulsi-Namla. This suggests that the parent rocks (granite and gneiss) of sericite schists had different proportions of mafic and felsic components. Among the alkali trace elements Rb shows good correlation with $\mathrm{K}_{2} \mathrm{O}$ in all the three areas, indicating the addition of $\mathrm{Rb}$ during $\mathrm{K}$-metasomatism.

The chondrite normalized (normalization values after Taylor and McLennan 1985) as well as parent gneiss/granite normalized REE patterns for the sericite schists are given in figure 10(a to f). In the case of Tulsi-Namla the REE composition of the biotite gneiss (sample \# D1S1) has been used for the normalization, and for the samples from Madar and Barodia, the composition of Ahar River Granite has been used. REE patterns of samples from all the three areas are similar and are 'U'-shaped because of large LREE fractionation and HREE enrichment. Compared to the parent gneiss and granite, the HREE in sericite schist samples is found to be enriched (see figure 10a,c,e). This becomes very clear in the parent rock normalized patterns (figure 10b,d,f). While in most of the samples from all the three areas LREE are depleted relative to the parent rock, their HREE show an enrichment of 3 to 5 times. Most of the samples yield negative $\mathrm{Eu}$ anomalies when normalized to basement rock compositions (Madar samples - 0.55 to 0.91 ; Barodia samples -0.45 to 1.47 ; Tulsi-Namla samples - 0.62 to 1.72 ).

The Ahar River Granite normalized REE patterns for serial samples of Barodia reveals an interesting story. The samples with moderate $\mathrm{Al}_{2} \mathrm{O}_{3}$ content (\#s B to R4/6) being close to the contact with parent granite show very low $(\mathrm{Gd} / \mathrm{Yb})_{\mathrm{N}}$ values $(0.12$ to 0.26$)$, while the sample collected near the upper contact (\# W4/6), which has maximum $\mathrm{Al}_{2} \mathrm{O}_{3}$ content (c. $37 \%$ ), has highest value of 0.55 . The $\mathrm{Al}_{2} \mathrm{O}_{3}$ contents in these serial samples show good correlation with the Ahar River Granite normalized $(\mathrm{Gd} / \mathrm{Yb})$ values (figure 11). Average $\mathrm{Al}_{2} \mathrm{O}_{3}$ and $(\mathrm{Gd} / \mathrm{Yb})_{\mathrm{N}}$ values of samples from Tulsi-Namla and Madar are also plotted in figure 11. It can be noticed that their $(\mathrm{Gd} / \mathrm{Yb})_{\mathrm{N}}$ is higher relative to the Barodia samples having moderate $\mathrm{Al}_{2} \mathrm{O}_{3}$.

Whole-rock normalized REE patterns for kyanites and sericites separated from samples of Madar are (\# C4-1; G4-1) shown in figure 12. The HREE in kyanite separate is enriched with respect to the whole rock indicating that the HREE enrichment is a premetasomatic feature. The sericite separates show REE patterns similar to the whole-rock indicating that sericitization of kyanites is nearly complete. However, they show negative Eu anomalies when normalized with the whole-rock. This suggests that the metasomatic alteration took place probably under reducing conditions.

\section{Discussion}

In metamorphic terranes identification of the original nature of any rock should especially consider the aspect of stratigraphy (Billings 1950). The consistent 


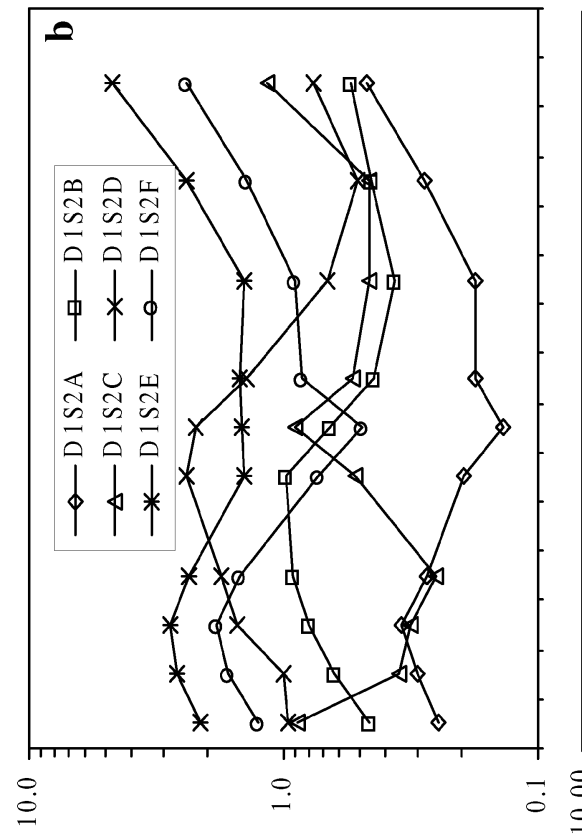

SS!วUD/Yวоบ

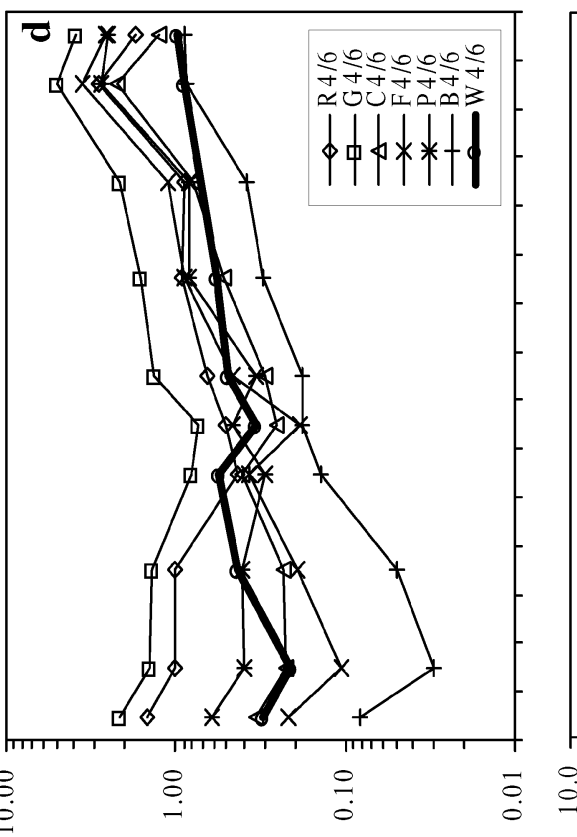

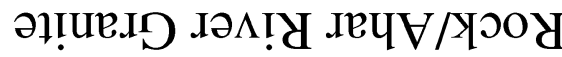

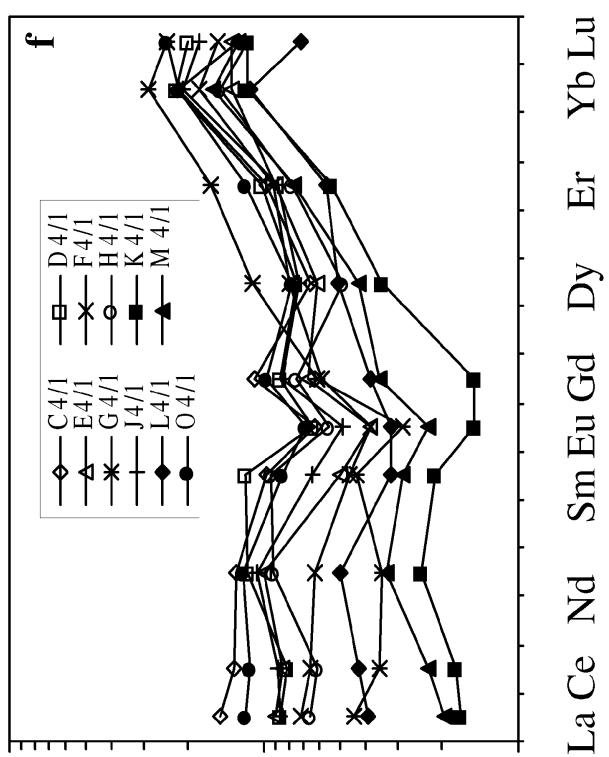

$\stackrel{\circ}{-}$

تี
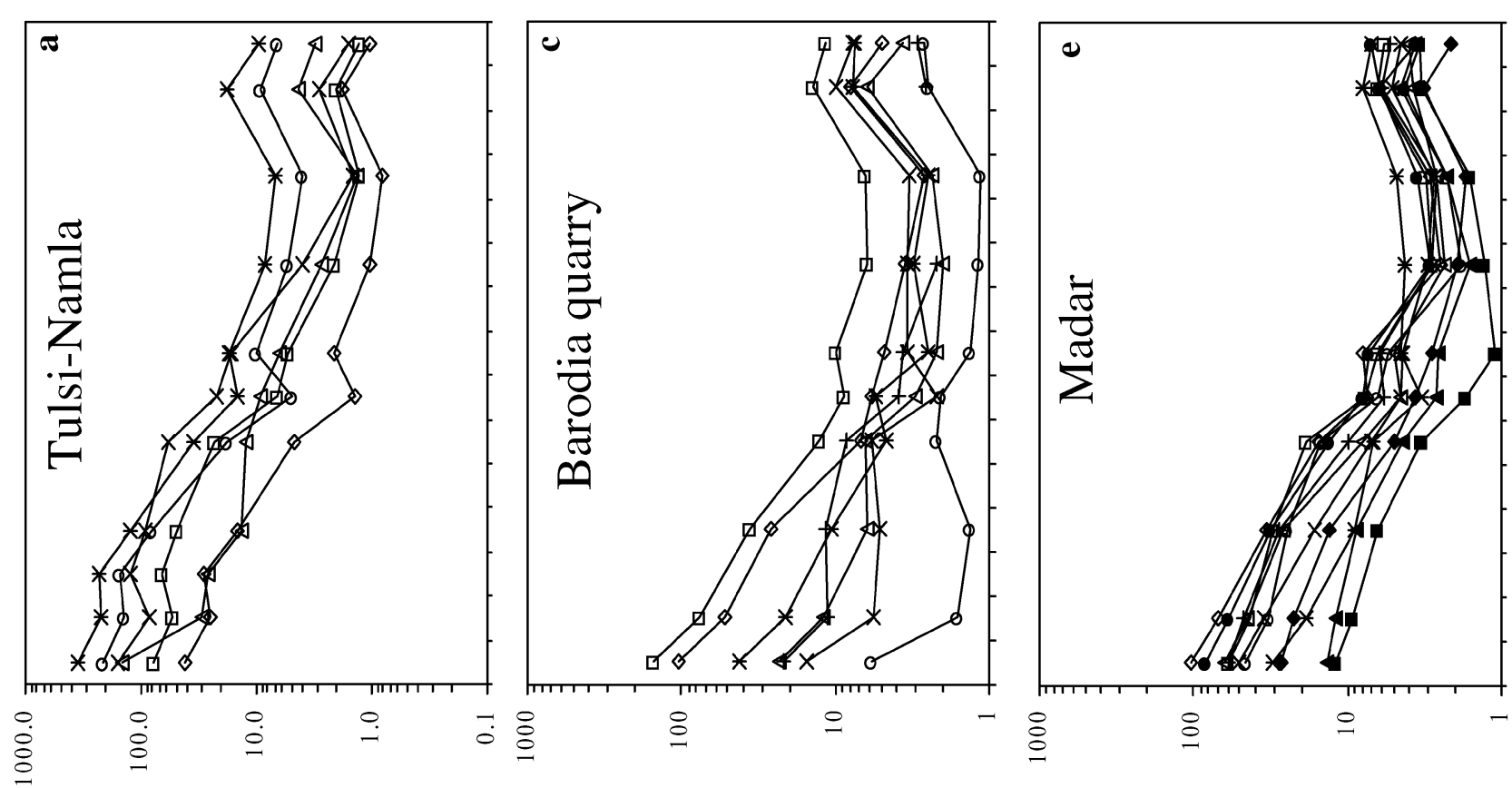

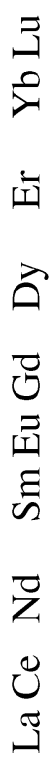

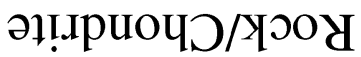




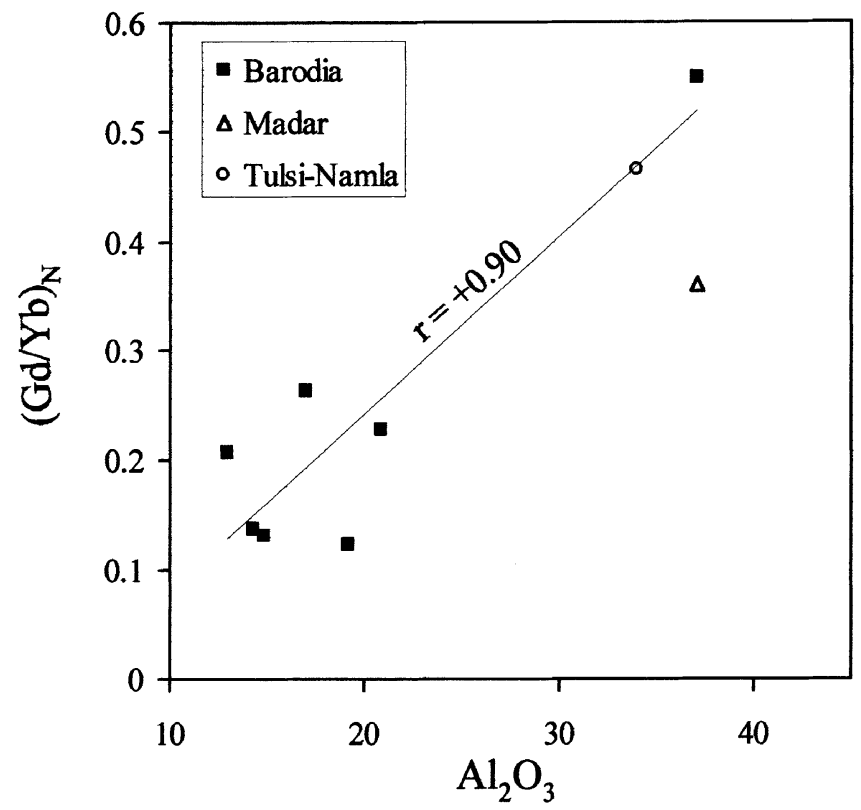

Figure 11. $\quad \mathrm{Al}_{2} \mathrm{O}_{3}$ vs. $(\mathrm{Gd} / \mathrm{Yb})_{\mathrm{N}}$ (Ahar River Granite normalized values) of sericite schists. The samples from Barodia exhibit positive correlation. The average values for the TulsiNamla and Madar areas are also plotted.

occurrence of the sericite deposits always at the interface of the Archean MGC and the Proterozoic Aravalli Supergroup points to the fact that they represent a well-defined stratigraphic horizon, a feature common to paleosols. It has been suggested by many workers that the major stratigraphic boundaries in the Precambrian terranes are the locales for paleosols (Retallack et al 1984; Retallack 1990; Kimberley and Holland 1992). Such unconformitybound paleosols have been recognized in many Precambrian terranes (Prasad and Roscoe 1991, 1996; Gay and Grandstaff 1980; Grandstaff et al 1986). Apart from their restricted occurrence at the basement-cover sequence interface, the upper sharp and lower gradational contact of the sericite schists of Rajasthan conforms to the field criteria for recognizing paleosols (see Grandstaff et al 1986). Occurrence of clasts of these sericite schists in the overlying conglomeratic quartzites further corroborates the above inference (cf. Rye and Holland 1998). The observable color variations due to mineralogical variations in sericite schists are consistent with a paleosol origin for these rocks. Further, the possibility that these rocks are products of shearing can be ruled out based on the fact that they occur independent of shear zones.
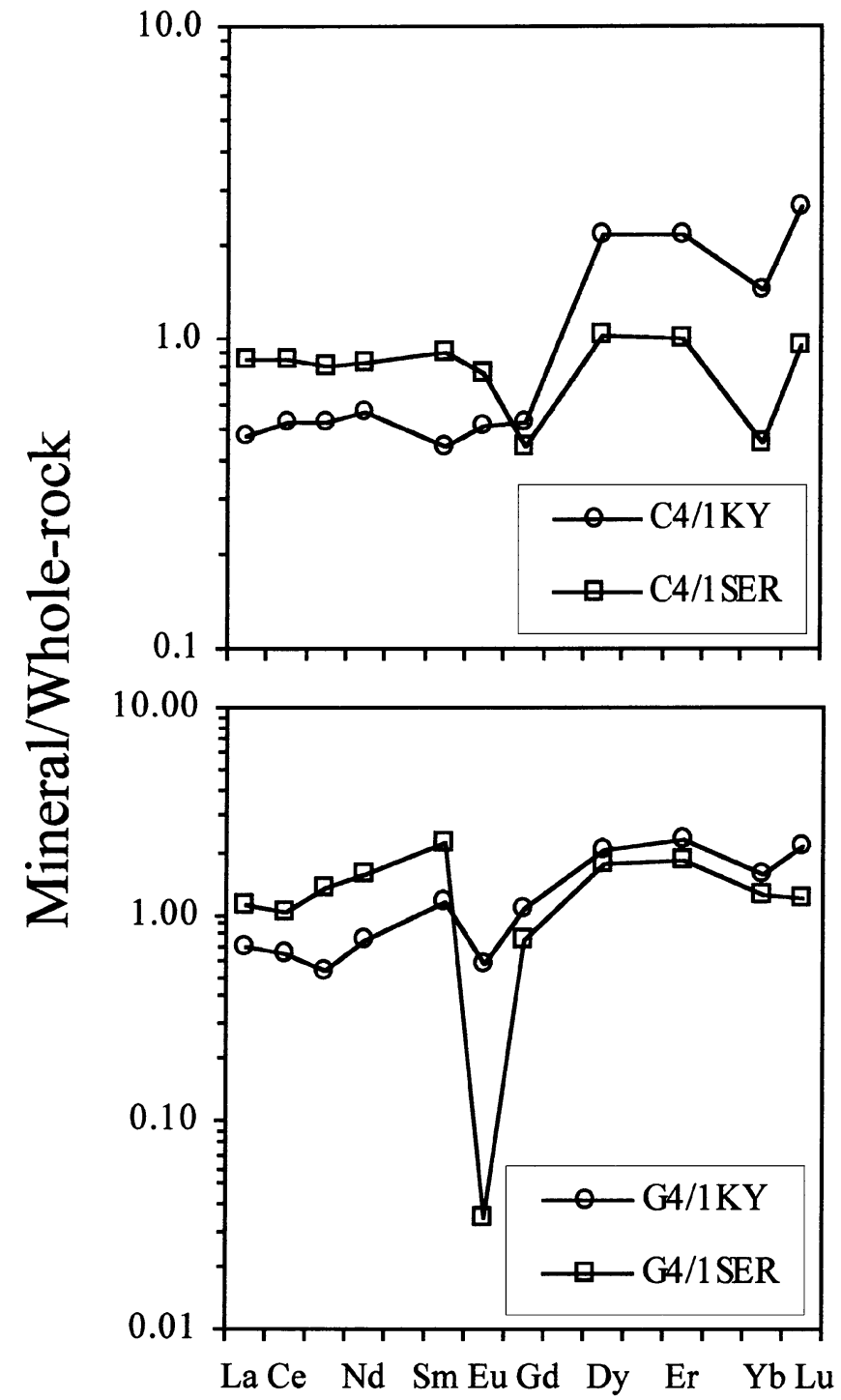

Figure 12. Whole-rock normalized REE patterns of kyanite and sericite minerals separated from sample \#s C4/1 and G4/1 of Madar. Note the negative Eu anomalies in sericite separates.

Petrographic studies show that the zone, which can be demarcated as paleosol exhibits a chlorite-quartzsericite rich layer at the base and a sericite-rich horizon towards the top. One common feature that distinguishes this horizon is the invariable presence of relict kyanite grains in it. The granites/gneisses beneath the sericite schist zone and the quartzites overlying it are devoid of any kyanite. Zircons in the sericite schists are euhedral and do not provide any evidence of transport. Also the morphology of these zircons resembles those in the underlying gneisses and

Figure 10. Chondrite, parent rock (gneiss/granite) normalized REE patterns of sericite schists. All chondrite normalized REE patterns (a,c,e) are ' $U$ ' shaped because of enrichment of both LREE and HREE. The parent rock normalized REE patterns (b,d,f) exhibit LREE depletion and HREE enrichment. Among the serial samples of Barodia, the sample close to the upper contact (\#W4/6 - depicted with the bolder line in figure $9 \mathrm{~d}$ ) show a flat HREE pattern having a higher $(\mathrm{Gd} / \mathrm{Yb})_{\mathrm{N}}$ value. 
granites. This coupled with gradational contact between sericite zone and the gneiss/granite, and also the relict granitic textures preserved in the chloritequartz-sericite layer, suggests paleosol nature for the protoliths of the sericite schists.

The formation of sericites from plagioclase in gneisses and granitoids, and from the kyanites in sericite schists coupled with the $\mathrm{K}$ enriched nature of the volcanic rocks of the Delwara Formation (Sreenivas et al 1999), which immediately overlies the paleosol horizon-all indicate that sericitization (K-metasomatism) is a pervasive, post-Aravalli process. The compositions of sericites that have been formed from metasomatic alteration of feldspars of gneisses are found to be different from the sericites of these deposits which have been formed from the breakdown of kyanites (see figure 5a,b). It therefore can be inferred that the sericitic mica in these deposits has formed due to the metasomatic alteration of kyanites and not from feldspars of the gneisses.

The major element compositions of sericite schists show $\mathrm{Al}_{2} \mathrm{O}_{3}$ enrichment and depletion in $\mathrm{SiO}_{2}, \mathrm{FeO}$ (tot), $\mathrm{MnO}, \mathrm{MgO}, \mathrm{CaO}$ and $\mathrm{Na}_{2} \mathrm{O}$ contents and are consistent with the changes observed during chemical weathering as established long ago by Ebelman (1845). Progressively upward enrichment in $\mathrm{Al}_{2} \mathrm{O}_{3}$ in the serial samples of Barodia quarry are similar to changes associated with weathering. Samples from all the three areas are characterized by very high $\mathrm{Al}_{2} \mathrm{O}_{3}$ contents ( $>35 \%$ ), a feature which is typical of paleosols. The serial samples of Barodia show good correlation between $\mathrm{Al}_{2} \mathrm{O}_{3}$ and $\mathrm{TiO}_{2}$ similar to many Precambrian paleosols, which show welldefined characteristics of paleosols (cf. Sreenivas and Srinivasan 1994). The compositional gap observed in the $\mathrm{Al}_{2} \mathrm{O}_{3}$ vs. $\mathrm{TiO}_{2}$ diagram (figure 7 ) can be related to transformation of illite/smectite to kaolinite during progressive weathering. Limited deviations in the immobile elemental ratios such as $\mathrm{Ti} / \mathrm{Al}$ and $\mathrm{Ti} / \mathrm{Zr}$ of sericite schist samples from the basement gneiss/ granite compositions are similar to the pattern observed in modern soils (cf. Maynard 1992).

While the foregoing major element geochemical evidences suggest a paleosol origin for sericite schists of Rajasthan, the anomalous $\mathrm{K}_{2} \mathrm{O}$ enrichment in them is not in accordance with changes accompanying chemical weathering. In fact anomalously high contents of $\mathrm{K}_{2} \mathrm{O}$ have been observed in many Precambrian paleosols and have been considered as very problematic in the identification of paleosols by several workers (cf. Retallack et al 1984; Palmer et al 1989; Kimberley and Holland 1992). The petrographic relations suggest that the sericitization of kyanites is a secondary event that can be related to potash metasomatism that has affected the protoliths of the sericite schists. Further, they suggest that the upward increase of $\mathrm{K}_{2} \mathrm{O}$ in the serial samples of Barodia is due to the variation in the abundance of sericite. It is well known that the degree of metasomatic alteration is controlled by the quantum and composition of the metasomatising fluids and the mineralogical composition of the rock undergoing alteration. In an environment of pervasive K-metasomatism as observed in the present case, it affects significantly the sodic plagioclases leaving the K-feldspars unaffected, as observed in the basement granites and gneisses. Similarly minerals rich in ferromagnesian constituents like chlorite, and quartz cannot be affected by K-metasomatic alteration, while it preferentially attacks Alsilicate minerals because of their high alumina and low alkali contents giving rise to sericite. Such a behavior explains the variation in the sericite content in the serial samples of the Barodia quarry, where the lower chlorite-quartz horizon has lower sericite content and the upper alumina-rich part has a higher sericite content. The metasomatic enrichment of $\mathrm{K}_{2} \mathrm{O}$ in the sericite schists has rendered their CIA values $(\sim 75)$ similar to normal shales instead of residual clays. However, their pre-metasomatic CIA values are very high, ranging from 85 to 100 . The residual clays listed by Pettijohn (1975) also show a range of CIA values between 85 and 100 (Nesbitt and Young 1982), indicating that the protoliths of the sericitic rocks might originally be products of intense chemical weathering.

The immobile trace elements, especially the HFSE exhibit good correlation among themselves. The REE patterns indicate an enrichment especially in the HREE relative to parent gneiss/granite, while the LREE remain grossly similar. The HREE also show fractionation so that their $(\mathrm{Gd} / \mathrm{Yb})_{\mathrm{N}}$ values are lower. The $(\mathrm{La} / \mathrm{Sm})_{\mathrm{N}}$ values of sericite schists are similar to the values of parent gneiss/granite. The HREE fractionation in serial samples of Barodia are similar to changes associated with weathering profiles. It can be seen from figure 11 that the samples representing the moderate or incipient weathering stage as indicated by moderate $\mathrm{Al}_{2} \mathrm{O}_{3}$ content have been more enriched in HREE than the one which has been altered most and occurs towards the top of the sericite schist zone. In a classical study, Nesbitt (1979) demonstrated exactly similar patterns of changes in REE compositions in a modern weathering profile that developed on the Torongo Granodiorite of Australia. He demonstrated that the HREE will be leached in the residual zones (highly weathered zones) because of lower $\mathrm{pH}$ conditions and will be precipitated in the moderately altered zones because of an increase in $\mathrm{pH}$. The HREE may form carbonate ligands and will get mobilized in the residual zones of a soil profile. It can also be noticed that the samples with high $\mathrm{Al}_{2} \mathrm{O}_{3}$ contents from all the three regions have generally higher $(\mathrm{Gd} / \mathrm{Yb})_{\mathrm{N}}$ values when compared to the samples having moderate $\mathrm{Al}_{2} \mathrm{O}_{3}$ contents, suggesting that the HREE fractionation in the sericite 
schist samples is largely controlled by chemical weathering.

The $\mathrm{Zr}$ considered to be immobile shows good correlation with $\mathrm{Al}_{2} \mathrm{O}_{3}$ in the Tulsi-Namla samples, as would be in the case of a weathering process. The lack of good correlation between $\mathrm{Al}_{2} \mathrm{O}_{3}$ and $\mathrm{Zr}$ in the case of serial samples of Barodia is due to the $\mathrm{Zr}$ enrichment in the middle portions of the paleosol profile. Such an enrichment has been attributed to the unequal distribution of zircon in the parent rock and immobility of $\mathrm{Zr}$ during weathering. It may be mentioned here that Nesbitt (1979) has also observed $\mathrm{Zr}$ enrichment in incipiently weathered sample of the modern weathering profile developed on Torongo Granodiorite.

The foregoing discussion clearly shows that the field, petrographic and geochemical attributes of the sericite schists of Rajasthan meet the criteria put forth recently by Rye and Holland (1998) for identifying a definite paleosol.

\subsection{Implication of iron content of Aravalli paleosols on the atmospheric $\mathrm{PO}_{2}$ evolution}

The Aravalli paleosols developed between 2.5 and 2.1 Ga. According to Ohmoto's (Ohmoto 1996) classification of Precambrian paleosols, the Aravalli paleosols reflect characters of M-type and H-type paleosols. The presence of (a) upper sericite and lower chlorite zone, (b) increase in $\mathrm{Fe}^{3+} / \mathrm{Ti}$ ratios relative to the parent rocks, and, (c) depletion of iron in sericite-rich upper zones and its enrichment in the lower chloritic zones indicates that they may be similar to M-type paleosols. However, the good correlation between $\mathrm{Fe}^{2+} / \mathrm{Ti}$ and $\mathrm{Fe}^{3+} / \mathrm{Ti}$ indicate that they may be similar to H-type paleosols. From the foregoing it may be suggested that there has been a remobilization of Fe probably associated with K-metasomatism which has taken place under reducing conditions as indicated by the negative $\mathrm{Eu}$ anomalies in sericite separates (figure 12). Estimation of gain or loss of iron in these paleosols relative to the parent rock due to weathering alone may not be possible. However, development of $\mathrm{Al}_{2} \mathrm{O}_{3}$-rich and Fe-poor paleosols could be envisaged for the Aravalli paleosols, because even chlorite-quartz-sericite zone at the base has iron less than the parent granite.

Rye and Holland (1998) have observed that the Precambrian paleosols $<2.2 \mathrm{Ga}$ are iron enriched. This led them to estimate the increase in the $\mathrm{PO}_{2}$ of atmosphere from $\leq 0.002$ atm to $\geq 0.03$ atm some time during 2.2 to $2.0 \mathrm{Ga}$. Oxygenation of atmosphere is known to be associated with ${ }^{13} \mathrm{C}$ enrichment in carbonate carbon on a global scale for the first time in the earth's history around 2.22 to $2.06 \mathrm{Ga}$ (Karhu and Holland 1996). Paleosols developed prior to this event are considered to have developed in oxygen-deficient environment. The occurrence of ${ }^{13} \mathrm{C}$ enriched carbo- nate rocks overlying the iron-deficient paleosols have been observed in several continents such as northern Europe and north America (Ojakangas et al 1998) as well as South Africa (Buick et al 1998). Such a ${ }^{13} \mathrm{C}$ excursion in carbonate rocks has been documented from Jhamarkotra Formation of the Lower Aravalli Supergroup, which overlies the paleosols by Sreenivas et al $(1998,1999,2001)$. Therefore, it is suggested that the $\mathrm{Al}_{2} \mathrm{O}_{3}$ enriched Aravalli paleosols must have developed in oxygen-deficient atmosphere. It is, however, possible that the observed negative $\mathrm{Eu}$ anomalies in the parent gneiss/granite normalized REE patterns of most of the samples are the combined effect of the protolithic characteristic as well as the activity of reducing metasomatic fluids that brought about sericitization.

\section{Conclusions}

Based on detailed field, petrographic and geochemical studies, the following sequence of events for the formation of the sericite deposits of Udaipur region is envisaged:

- The cratonic basement of the Aravalli Supergroup which stabilized at $\sim 2.5 \mathrm{Ga}$ (Wiedenbeck et al 1996), was subjected to intense chemical weathering up to $\sim 2.1 \mathrm{Ga}$, the dawn of Aravalli sedimentation. This led to the formation of soils consisting of $\mathrm{Al}_{2} \mathrm{O}_{3}$-rich clays.

- Apparently these soils were converted to pyrophyllites during diagenesis and some of the relicts of pyrophyllites in small microscopic patches in the sericite deposits of Madar may represent this phase.

- These diagenetically altered high- $\mathrm{Al}_{2} \mathrm{O}_{3}$ soils suffered metamorphism to give rise to kyanite schists.

- Subsequently, a post-Aravalli potash-metasomatic event altered the metamorphic assemblage to sericite schists.

\section{Acknowledgements}

The present work is a part of the study on the Archean-Proterozoic boundary in Rajasthan, India funded by the Department of Science and Technology and the doctoral dissertation of BS. BS is supported by a Research Fellowship of the Council of Scientific and Industrial Research. Authors acknowledge fruitful discussions with Dr. B S Paliwal and Dr. S Das Sharma. They are thankful to Dr. Harsh K Gupta, Director, NGRI for encouragement and permission to publish this work. They also acknowledge the help of Drs. V Balaram, P K Govil, and R Natarajan of NGRI in the analytical work. Critical comments by two anonymous reviewers helped in the improvement of the paper. 


\section{References}

Ahmad T and Rajamani V 1988 Geochemistry and petrogenesis of mafic inclusions in Banded Gneissic Complex, near Nathadwara: implications to BGC - Aravalli relationships; In Precambrian of Aravalli Mountain, Rajasthan, India (ed) A B Roy Geol. Soc. India Mem. 7 pp. 327-340

Balaram V, Ramesh S L and Anjiah K V 1996 New trace element and REE data in thirteen GSF reference samples by ICP-MS; Geostand. News Lett. 20 71-78

Banerjee D M 1996 A Lower Proterozoic paleosol at BGC Aravalli boundary in south-central Rajasthan, India; $J$. Geol. Soc. India $\mathbf{4 8}$ 277-288

Barrientos X and Selverstone J 1987 Metamorphosed soils as stratigraphic indicators in deformed terranes: An example from the Eastern Alps; Geology 15 841-844

Barrientos X and Selverstone J 1988 Reply "Metamorphosed soils as stratigraphic indicators in deformed terranes: An example from the Eastern Alps"; Geology 16572

Bence A E and Albee A L 1968 Empirical correction factors for electron probe microanalysis of silicates and oxides; J. Geol. 76 382-403

Billings M P 1950 Stratigraphy and the study of metamorphic rocks; Bull. Geol. Soc. America 61 435-448

Buick I S, Uken R, Gibson R L and Wallmach T 1998 High- $\delta{ }^{13} \mathrm{C}$ Paleoproterozoic carbonates from the Transvaal Supergroup, South Africa; Geology 26 875-878

Chauhan D S 1970 Pyrophyllite deposit of Barara village, Udaipur district, Rajasthan; Bull. Indian. Geol. Assoc. 39-12

Dash B, Sahu K N and Bowes D R 1987 Geochemistry and original nature of Precambrian khondalites of Eastern Ghats, Orissa, India; Trans. Royal Soc. Edinburgh: Earth Sciences 78 115-127

Deb M 1999 Metallic mineral deposits of Rajasthan; In Proc. Sem. on Geology of Rajasthan Status and Perspective (ed) P Kataria (Geology Dept. MLSU, Udaipur, India) pp. 213-237.

Ebelman J J 1845 Sur les produits de la decomposition des especes minerales de la famille des silicates; Am. des Mines. 7 $3-66$

Feakes C R, Holland H D and Zbinden E A 1989 Ordovician paleosols at Ariasag, Nova Scotia, and the evolution of the atmosphere; In Paleopedology-Nature and applications of paleosols (eds.) A Bronger and J Catt, Catena Supplement 16 (Cremlingen) pp. 207-232

Fedo C M, Nesbitt H W and Young G M 1995 Unravelling the effects of potassium metasomatism in sedimentary rocks and paleosols, with implications for paleoweathering conditions and provenance; Geology 23 921-924

Gay A L and Grandstaff D E 1980 Chemistry and mineralogy of Precambrian paleosols at Elliot Lake, Ontario, Canada; Precamb. Res. 12 349-373

Golani P R 1989 Sillimanite-Corundum deposits of Sonapahar, Meghalaya, India: A metamorphosed Precambrian paleosol; Precamb. Res. 43 175-189

Gopalan K, Macdougall J D, Roy A B and Murali AV 1990 Sm-Nd evidence for 3.3 Ga old rocks in Rajasthan, northwestern India; Precamb. Res. 48 287-297

Grandstaff D E, Edelman M J, Foster R W, Zbinden E A and Kimberley M M 1986 Chemistry and mineralogy of Precambrian paleosols at the base of the Dominion and Pongola Groups (Transvaal, South Africa); Precamb. Res. 32 97-131

Guha D B and Garkhal R S 1993 Early Proterozoic Aravalli metasediments and their relation with the Ahar River Granite around Udaipur, Rajasthan; J. Geol. Soc. India 42 327-335

Harnois L 1988 The CIW index : a new chemical index of weathering; Sed. Geol. 55 319-322
Holland H D 1984 The chemical evolution of atmosphere and oceans (Princeton Univ. Press, Princeton N.J.) $582 \mathrm{p}$

Holland H D 1994 Early Proterozoic atmospheric change; In Early Life on Earth. Noble Symposium (ed) S Bengston $\mathbf{8 4}$ (Columbia Univ. Press, New York) pp. 237-244

Holland H D and Zbinden E A 1988 Paleosols and the evolution of atmosphere: Part I. In Physical and Chemical weathering in Geochemical cycles (eds) A Lerman and M Meybeck (Reidel: Dordrecht) pp. 61-82

Holland H D and Beukes N J 1990 A paleoweathering profile from Griqualand west, South Africa : evidence for a dramatic rise in atmospheric oxygen between 2.2 and 1.8 b.y B.P; $\mathrm{Am}$. J. Sci. 290 1-34

Holland H D and Rye R 1997 Evidence in pre-2.2 Ga paleosols for the early evolution of atmospheric oxygen and terrestrial biota; Comment Geology 25 857-858

Holland H D, Feakes C R and Zbinden E A 1989 The Flin Flon paleosol and the composition of the atmosphere 1.8 b.y. B.P; Am. J. Sci. 289 362-389

Kallioski J 1975 Chemistry and mineralogy of Precambrian paleosols in northern Michigan; Geol. Soc. Am. Bull. 86 371-376

Kallioski J 1977 Reply "Chemistry and mineralogy of Precambrian paleosols in northern Michigan"; Geol. Soc. Am. Bull. 881376

Karhu J A and Holland H D 1996 Carbon isotopes and the rise of atmospheric oxygen; Geology 24 867-870

Kimberley M M and Holland H D 1992 Introduction to Precambrian weathering and paleosols; In Early organic evolution - Implications for mineral and energy resources (eds) M Schidlowski, S Golubic, M M Kimberley, D M McKirdy and P A Trudinger (Berlin: Springer-Verlag) pp. 9-15

Lewan M D 1977 Comment "Chemistry and mineralogy of Precambrian paleosols in northern Michigan"; Geol. Soc. Am. Bull. 881375

Macfarlane W A, Danielson A and Holland H D 1994 Geology, major and trace element chemistry of Late Archean weathering profiles in the Fortescue Group, Western Australia: Implications for atmospheric $\mathrm{PO}_{2} ;$ Precamb. Res. 65 297-317

Maynard J B 1992 Chemistry of modern soils as a guide to interpreting Precambrian paleosols; J. Geol. 100 279-289

Nesbitt H W 1979 Mobility and fractionation of rare earth elements during weathering of a granodiorite; Nature $\mathbf{2 7 9}$ 206-210

Nesbitt H W and Young G M 1982 Early Proterozoic climates and plate motions inferred from major element geochemistry of Lutites; Nature 299 715-717

Ohmoto H 1996 Evidence in pre-2.2 Ga paleosols for the early evolution of atmospheric oxygen and terrestrial biota; Geology 24 1135-1138

Ohmoto H 1997 Reply "Evidence in pre-2.2 Ga paleosols for the early evolution of atmospheric oxygen and terrestrial biota"; Geology 25 858-859

Ojakangas R W, Marmo J S and Heiskanen K I 1998 Early Proterozoic glaciation, paleosol formation and deposition of orthoquartzites: On separate continents or on a supercontinent? Int. Symp. on Paleoclimates and the Evolution of Paleogeographic environments in the Earth's Geological History Petrozavodsk Russia p. 42

Palmer J A, Philips G N and McCarthy T S 1989 Paleosols and their relevance to Precambrian atmospheric composition; J. Geol. 97 77-92

Pettijohn F J 1975 Sedimentary rocks; (New York: Harper and Row Publ) $682 \mathrm{p}$

Pinto J P and Holland H D 1988 Paleosols and the evolution of the atmosphere, part II. In Paleosols and weathering through geologic time (eds) J Reinhardt and W Sigleo Spec. Pap. Geol. Soc. America 21-34 
Prasad N and Roscoe S M 1991 Profiles of altered zones at ca $2.45 \mathrm{Ga}$ unconformities beneath Huronian strata, Elliot Lake, Ontario: evidence for early aphebian weathering under anoxic conditions; Geol. Surv. Canada Paper 91-1c $43-54$

Prasad N and Roscoe S M 1996 Evidence of anoxic to oxic atmospheric change during 2.45-2.22 Ga from lower to upper sub-Huronian paleosols, Canada; Catena 27 105-121

Rahman A and Zainuddin S M 1990 Geochemistry and genesis of Ahar River Granite, northwest of Udaipur city, Rajasthan; J. Geol. Soc. India 35 620-630

Retallack G J, Kimberley M M and Grandstaff D E 1984 The promise and problems of Precambrian paleosols; Episodes $\mathbf{7}$ $8-12$

Retallack G J 1990 Soils of the past-An introduction to paleopedology (Boston: Unwin and Heyman Inc.) $520 \mathrm{p}$

Roy A B 1988 Stratigraphic and tectonic framework of the Aravalli Mountain Range. In: Precambrian of the Aravalli Mountain Rajasthan, India, (ed) A B Roy Mem. Geol. Soc. India 7 pp. $3-31$

Roy A B and Paliwal B S 1981 Evolution of lower Proterozoic epicontinental deposits: Stromatolite-bearing Aravalli rocks of Udaipur, Rajasthan, India; Precamb. Res. 14 49-74

Roy A B and Kroner A 1996 Single zircon evaporation ages constraining growth of the Aravalli craton, northwestern Indian shield; Geol. Mag. 133 333-342

Roy A B, Golani P R and Bejarniya B R 1985 The Ahar River Granite, its stratigraphic and structural relations with early Proterozoic rocks of south-eastern Rajasthan; J. Geol. Soc. India 26 315-325

Rye R and Holland H D 1998 Paleosols and the evolution of atmospheric oxygen: Critical review; Am. J. Sci. 298 $621-672$

Sharma R P 1979 Origin of pyrophyllite-diaspore deposits of the Bundelkhand Complex, central India; Mineral. Deposita 14 343-352

Sreenivas B and Srinivasan R 1994 Identification of paleosols in the Precambrian metapelitic assemblages of Peninsular
India - A major element geochemical approach; Curr. Sci. 67 89-94

Sreenivas B and Govil P K 1997 A method for the analysis of aluminium and potassium-rich silicate rocks by X-ray fluorescence spectrometry using synthetic calibration standards; J. Indian Chem. Soc. 74 742-744

Sreenivas B, Balaram V and Srinivasan R 1994 Trace and rareearth element contamination during routine preparation of sample powders for geochemical studies: Effects of grinding tools; Indian J. Geol. 66 296-304.

Sreenivas B, Das Sharma S, Zachariah J K, Kumar B, Patil D J, Padmakumari V M and Srinivasan R 1998 Evidence for the Lomagundi event in the Paleoproterozoic Aravalli Supergroup, NW India; Proc. of 9th Intl. Conf. on Geochronology, Cosmochronology and Isotope Geology (Beijing, China) p 122

Sreenivas B, Srinivasan R and Roy A B 1999 Geochemical changes across the Archean-Proterozoic Boundary - A study from the Udaipur area of Aravalli Mountain Belt, Rajasthan, India; In Proceedings of the Seminar on Geology of Rajasthan - Status and Perspective (ed) P Kataria (Geology Dept. M L Sukhadia Univ. Udaipur, India) pp. 57-86

Sreenivas B, Das Sharma S, Kumar B, Patil D J, Roy A B and Srinivasan R 2001 Positive $\delta^{13} \mathrm{C}$ excursion in carbonate and organic fractions from the Paleoproterozoic Aravalli Supergroup, northwestern India; Precamb. Res. 106 277--290

Taylor S R and McLennan S M 1985 The continental crust: its composition and evolution (Oxford: Blackwell Sci. Publ.) $311 \mathrm{p}$

Wiedenbeck M, Goswami J N and Roy A B 1996 Stabilization of the Aravalli Craton (northwestern India) at $2.5 \mathrm{Ga}$ : Evidence from Ion Microprobe Zircon ages; Chem. Geol. 129 325-340

Williams F 1988 Comment "Metamorphosed soils as stratigraphic indicators in deformed terranes: an example from the eastern Alps"; Geology 16573

Zbinden E A, Holland H D, Feakes C R and Dobes S K 1988 The Sturgeon Falls paleosols and the composition of atmosphere 1.1 b.y. B.P; Precamb. Res. 42 141-163 